\title{
Disk populations from HIPPARCOS kinematic data
}

\section{Discontinuities in the local velocity distribution $\star$}

\author{
S. Alcobé and R. Cubarsi
}

Dept. Matemàtica Aplicada IV, Universitat Politècnica de Catalunya, 08034-Barcelona, Catalonia, Spain

e-mail: rcubarsi@mat.upc.es

Received 3 June 2005 / Accepted 26 July 2005

\begin{abstract}
The full space motions - including radial velocities - of a stellar sample drawn from HIPPARCOS catalogue are used to discriminate differentiated statistical behaviours that are associated with stellar populations in the solar neighbourhood. A sampling parameter is used to build a hierarchical set of nested samples, where a discontinuous pattern, based in a partition introduced by two normal distributions, scans the subsamples. Two quantities inform whether any subsample fits properly into the discontinuous model. A $\chi^{2}$ test measures the Gaussianity of both components, and the entropy of the mixture probability gives account of how informative the resulting segregation is. The less informative partition is the one with maximum population entropy, which provides most representative kinematic parameters. Each new population merged to the cumulative subsample produces a discontinuity in the plot entropy versus sampling parameter, that allows to determine the number of populations contained in the whole sample. The resulting method has been named MEMPHIS, Maximum Entropy of the Mixture Probability from HIerarchical Segregation.

In addition to both main kinematic components, thin and thick disk, with respective velocity dispersions $(28 \pm 1,16 \pm 2,13 \pm 1)$ and $(65 \pm 2,39 \pm$ $9,41 \pm 2) \mathrm{km} \mathrm{s}^{-1}$, two discrete non-Gaussian subcomponents are detected within the thin disk. These populations are identified with early-type and young disk stars. Moreover, a continuous old disk population is mixed with the foregoing subcomponents composing all together the thin disk. Older thin disk stars have a velocity dispersion overlapping a wing of the thick disk. Although they could appear like an intermediate continuous population, nested subsamples distributions allow us to conclude that they definitively belong to the thin disk, and that a clear discontinuity detaches thick from thin disk. Almost the same qualitative results, but with less accuracy, are obtained whether MEMPHIS is applied to subsamples from the Third Catalogue of Nearby Stars (CNS3).

A dynamic model according to Chandrasekhar's approximation, under particular symmetry hypotheses, allows to interpret the results. The non-vanishing vertex deviation - lower for older stars - of all Galactic components is suggesting that, at least, point-axial symmetry is required in order to explain the local kinematic behaviour. According to this model, the oldest thick disk population, with no net radial movement, can be extrapolated, having heliocentric velocities of $-76 \pm 2 \mathrm{~km} \mathrm{~s}^{-1}$ in rotation, and $-18 \pm 1 \mathrm{~km} \mathrm{~s}^{-1}$ in the radial direction. Early-type stars show a worthy local singularity, nearly with no net radial motion, similarly to the oldest thick disk stars. Older populations - half of the thin disk and the whole thick disk - share a common differential galactic movement, suggesting a common dynamical origin for the rupture of the axial symmetry.

The relationship between the maximum stellar velocity of a sample and its average age $\tau$ is discussed, finding an approximate relation $|\boldsymbol{V}|_{\max } \propto \tau$. Local stellar populations can be described from a Titius-Bode-like law for the radial velocity dispersion, $\sigma_{1}=6.6\left(\frac{4}{3}\right)^{x}$, so that for natural values $x=2,3,5,8$ it determines average energy levels of discrete populations, while for continuous intervals $x \leq 5$ and $x \geq 7$ it describes the velocityage evolution of thin and thick disk components, according to $x \sim 1.5 \ln \tau$.
\end{abstract}

Key words. stars: kinematics - stars: Population II - methods: analytical - Galaxy: disk

\section{Introduction}

Stars in the solar neighbourhood are generally associated with Galactic components. Several works on stellar populations (e.g. Wyse \& Gilmore 1995) confirm the presence of three main populations: thin disk, thick disk and halo. Nevertheless, since Baade's work in 1944, the idea of stellar populations has

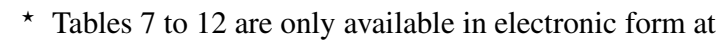
http://www . edpsciences.org largely evolved. Nowadays a stellar population can be defined as a group of stars that have a similar and regular set of properties in some subset of a multi-dimensional parameter space. In general, spatial distribution and kinematics allow to define distinct morphologic components, while age and abundance describe other populations within those components (King 1995).

From a kinematic viewpoint, a common working hypothesis is to assume the velocity density function of each single stellar component as Gaussian. For example Ojha et al. (1996) 
assume that in a particular volume of the Galaxy there exists a finite mixture of discrete stellar populations, and such populations are modelled as normal ones. Let us remember two advantages of such assumption. By one hand the Gaussian distribution is a particular solution of Chandrasekhar equations for statistical equilibrium (Chandrasekhar 1942), so that population parameters can be dynamically interpreted. On the other hand, from a bayesian viewpoint, the normal distribution is the one containing the maximum uncertainty compatible with known means and covariances and, therefore, it is well suited as a prior distribution.

In a previous paper (Cubarsi \& Alcobé 2004, hereafter Paper I) we had already identified thin and thick disk components in a local sample of HIPPARCOS Calatogue. However, between both kinematic components we had found some intermediate subcomponent, not being clear what population these stars belong to. In other words, the old question of whether there is or is not a continuous transition between thin and thick disks had arisen.

In the present paper we analyze in more detail the possible discontinuities of the stellar velocity distribution in the solar neighbourhood, by drawing a set of hierarchically selected samples from HIPPARCOS catalogue. Our technique is a combination of two separate procedures: a sample selecting filter and a segregation method. By continuously increasing an ideal sampling parameter, we build a set of nested subsamples that may contain an increasing number of populations. Then, a bimodal pattern is applied in order to identify some differentiated kinematic behaviour within the subsamples. The segregation may be done by any numerical algorithm that approximates the working sample as a mixture of two Gaussian components, giving also a measure of the plausibility of such approach, in our case the $\chi^{2}$ error. However, the Gaussianity of segregated components is not strictly required, since the model is used as a less informative prior hypothesis. We apply the algorithm described in Paper I, which works from the cumulants up to fourth order of the total input sample, although other strategies could be used in future works.

Once the subsample has been partitioned, the entropy of the mixture probability as a function depending on the sampling parameter, gives a plot that informs about the number of kinematic populations contained in the whole sample. This is based on the fact that the population entropy is a measure of how representative, less informative the segregation is. Thus, while selecting stars belonging to a population that already existed in a previous subsample, the entropy remains nearly constant, or at maximum relative levels. Alternatively, when enlarging the subsample with stars belonging to a new population, the plot shows a straight downward slope, or a discontinuity.

The complete method has been named MEMPHIS (Maximum Entropy of the Mixture Probability from HIerarchical Segregation). Therefore, entropy maxima inform us about number of populations, and values of $\chi^{2}$ measure the Gaussianity. It is worth noting that relative minimums of $\chi^{2}$ take place on intervals with relative maxima of population entropy, being the later a more efficient indicator.

It is well known that, in order to discriminate populations, some parameters like the velocity component perpendicular to the galactic plane, the rotational velocity component, and the modulus of the velocity have been used. For more than two populations, we found that the one closest to an ideal discriminating parameter is the absolute value of the total space motion, $|\boldsymbol{V}|$, referred to the cumulative local standard of rest of the nested subsamples (cumulative subcentroid). The properties of such an ideal sampling parameter will be discussed in next sections.

In general, we looked for stellar groups large enough as to be considered a population in the statistical sense, even they had not a normal distribution. In such a case, the Gaussian approach might give a sufficient good estimation of population parameters. Hereafter the non-Gaussian populations will be referred as subcomponents, while the Gaussian ones will be named kinematic components.

The method was applied to HIPPARCOS Catalogue (ESA 1997), with the whole velocity space obtained from Hipparcos Input Catalogue (ESA 1992) (see Paper I for details). The sample was limited to $300 \mathrm{pc}$, so that only stars belonging to Galactic disk were included, with a total of 13678 stars. In order to compare the improvement of the results in front of older catalogues, the Third Catalogue of Nearby Stars CNS3 (Gliese \& Jahreiß 1991) was also used.

The application of MEMPHIS to HIPPARCOS leads to the following stellar populations with the following velocities $\left(\mathrm{km} \mathrm{s}^{-1}\right)$ expressed in a cylindrical heliocentric coordinates system.

The main disk structure is supported by two stellar components, obtained from a maximum value of the sampling parameter $|\boldsymbol{V}|_{\max }=209 \mathrm{~km} \mathrm{~s}^{-1}$ :

- thin disk, with $91 \%$ stars of the overall sample, with dispersions $\left(\sigma_{1}, \sigma_{2}, \sigma_{3}\right)=(28 \pm 1,16 \pm 2,13 \pm 1)$, and vertex deviation $\varepsilon=10 \pm 2^{\circ}$;

- thick disk, with $9 \%$ of stars, $\left(\sigma_{1}, \sigma_{2}, \sigma_{3}\right)=(65 \pm 2,39 \pm$ $9,41 \pm 2), \varepsilon=7 \pm 3^{\circ}$.

Two subcomponents with non-Gaussian distribution are also found within the thin disk structure, for $|\boldsymbol{V}|_{\max }=51 \mathrm{~km} \mathrm{~s}^{-1}$. Both groups have a high deviation from Gaussianity in radial velocity, but not in other directions. Hence the following dispersions are estimations apart from a normal distribution context. The first subcomponent may be associated with early-type stars, with smaller dispersions, slower rotation, and a clear radial expansion. It is far from Stroemberg's law for the asymmetric-drift of disk stars, and it may be identified with the local Gould's belt structure. Instead, the other group, which is composed of young disk stars, is consistent with the asymmetric-drift relation of the whole thin disk.

- Early-type stars, about $37 \%$ of the overall sample, $\left(\sigma_{1}, \sigma_{2}, \sigma_{3}\right)=(12 \pm 31,11 \pm 1,7 \pm 1)$

- young disk stars, with $38 \%$ stars and $\left(\sigma_{1}, \sigma_{2}, \sigma_{3}\right)=(16 \pm$ $22,14 \pm 1,14 \pm 1)$.

Nevertheless, the subsamples limited by $|\boldsymbol{V}|_{\max }$ lower than $51 \mathrm{~km} \mathrm{~s}^{-1}$ are dominated by moving groups, that could not be assimilated to homogeneous statistical populations.

The remaining $16 \%$ of the thin disk is a continuous old disk population that spreads out as a wing of the young disk stars. Its 
radial velocity dispersion reaches values up to $\sigma_{1}=50$, while, for subsamples with $51 \leq|\boldsymbol{V}|_{\max } \leq 125$, the whole thin disk component increases as $16 \leq \sigma_{1} \leq 28$. However, the vertical dispersion remains nearly constant at $\sigma_{3}=13$.

The extreme old disk stars are mixed with a broad wing of the thick disk in the range $38 \leq \sigma_{1} \leq 50$, what simulates the existence of an intermediate population that would provide a continuous transition between thin and thick disks. However, as enlarging the subsamples, $|\boldsymbol{V}|_{\max } \leq 145$, the intermediate population is progressively reorganized, so that stars with smaller rotation and vertical velocities are included in the thin disk component, while a major number of stars builds up the thick disk component, having a ratio between vertical and rotation velocity dispersions around 1. For $|\boldsymbol{V}|_{\max }>145$ the thick disk is free from thin disk stars. These results are similar to those of Soubiran et al. (2003), although we have found vertex deviation also for old stars, which leads to reject the axial symmetry hypothesis for the local stellar components.

The axial symmetry can be relaxed by assuming a stellar system with point-to-point symmetry with respect to an axis, the one orthogonal to the galactic plane at the galactic center. This type of symmetry should be induced by a gravitational potential with similar features in opposite and equidistant points of the symmetry axis, allowing helicoidal and elliptic behaviours around this axis. If such a point-axial model is assumed, then the decreasing vertex deviation of older stars may be related with the radial and rotation mean velocities, so that the mean rotation galactocentric velocity for an extreme axisymmetric, steady-state thick disk, with zero radial mean velocity, can be extrapolated at $144 \pm 2 \mathrm{~km} \mathrm{~s}^{-1}$.

Therefore, working only from the velocity space of HIPPARCOS local sample we have got a portrait of the populations composing the local Galactic disk, that can be labeled according to their radial dispersions: Early-type stars $\left(\sigma_{1} \approx 12\right)$, young disk stars $\left(\sigma_{1} \approx 16\right)$, old disk population $\left(21 \leq \sigma_{1} \leq\right.$ 50 ), all together composing the thin disk component $\left(\sigma_{1} \approx 28\right)$. On the other hand, the local thick disk component has a value $\sigma_{1}=65 \mathrm{~km} \mathrm{~s}^{-1}$. Clear discontinuities have been found between early-type and young disk subcomponents, as well as between thin and thick disk components, while continuity of kinematic parameters takes place within old disk, thin disk and thick disk, as increasing the sampling parameter. Such characteristic radial dispersions can be described through a heuristic Titius-Bode-like law, $\sigma_{1} \approx 6.6\left(\frac{4}{3}\right)^{x}$, so that natural values of $x$ represent specific populations. Thus, if $x=0$ represents a hypothetic spherical population with $\sigma_{1}=\sigma_{2}=\sigma_{3}=6.6$ ( similar to the $z$-velocity dispersion of the earlier detected population), then early-type and young disk stars can be associated with $x=2$ and 3, the complete thin disk component with $x=5$, and the thick disk component can be assigned to a value $x=8$. These values could be associated with the average epicycle energy (e.g. Lacey 1984; Jenkins 1992) that disk populations reach in the secular heating process. Moreover, by using the age-velocity law describing the disk heating, we find that the sampling parameter $|\boldsymbol{V}|_{\max }$ and the age $\tau$ are approximately proportional within the thin disk, though the thick disk shows an similar trend. Also a relationship $x \sim 1.5 \ln \tau$ is estimated.
Finally, some conclusions about the differential movement of populations can be pointed out. A lag of rotation between both Galactic components of $51 \pm 3 \mathrm{~km} \mathrm{~s}^{-1}$, and in radial movement of $4 \pm 3 \mathrm{~km} \mathrm{~s}^{-1}$ is obtained, similarly than in Paper I. Early-type stars and young disk stars have a radial differential movement of $28 \pm 9 \mathrm{~km} \mathrm{~s}^{-1}$. Young and old disk stars, as well as thin disk, are moving in the same direction with respect to the thick disk. Hence, by referring such populations to the extrapolated oldest thick disk, with no net radial velocity, the mean radial galactocentric velocity of HIPPARCOS sample can be estimated as $\Pi_{0}=8 \pm 1 \mathrm{~km} \mathrm{~s}^{-1}$ toward the Galactic centre.

Our results are in good agreement with the analysis of Famaey et al. (2005) about local streams of dynamic origin, as well as the chemical and kinematic distribution of Milky Way's disk by Soubiran \& Girard (2005). In addition we have provided an answer to the question about the radial solar motion referred to a point with no net radial motion, which would correspond to the stars labeled as early-type, as well as the oldest thick disk stars.

The outline of the paper is as follows. The second section explains the statistical model. We review some basic concepts on stellar systems. In Sect. 2.1 the notation used for the mixture distribution model is defined, explicitly depending on the sample selecting parameter. In Sect. 2.2 the entropy of the mixture probability is introduced. In Sect. 2.3 we study requirements for an ideal sampling parameter in order to induce a hierarchy in the nested subsamples. In Sect. 2.4 entropy variations from a sample with three or more populations are analyzed. In Sect. 2.5 entropy variation curves are explained by using synthetic samples. In Sect. 2.6 the method is named.

The third section shows the application. HIPPARCOS sample is introduced in Sect. 3.1. In Sect. 3.2 the steps composing MEMPHIS algorithm are outlined. In Sect. 3.3, as a predictor step, the method is applied to HIPPARCOS heliocentric subsamples. In Sect. 3.4, as a corrector step, re-centred velocity subsamples are used. In Sect. 3.5 the number of populations composing the stellar sample is discussed. In Sect. 3.6 the results are summarized. In Sect. 3.7 MEMPHIS is applied to CNS3 catalogue, in order to compare results.

The fourth section is for discussion of results, where some general conclusions are summarized. In Sect. 4.1 some kinematic consequences are pointed out. In Sect. 4.2 the results are interpreted according to a Chandrasekhar model under pointaxial symmetry hypothesis. In Sect. 4.3 we compare our analysis with two recent studies of the small and large-scale structure of the velocity distribution. In Sect. 4.4 the sampling parameters and the population velocity dispersions are related to the age-velocity law of disk heating.

\section{Statistical model}

Let us assume that the Galactic system is composed of several stellar populations. According to Chandrasekhar's approach, if a population has reached the statistical equilibrium, its phase space density function $f(t, r, \boldsymbol{V})$ at time $t$, in the 
Table 1. Non-null means, central moments and vertex deviation in a galactocentric coordinate system, depending on symmetry hypotheses, for one Gaussian population, under the assumption of a symmetry plane $z=0$. Time dependency for point-axial systems introduces only qualitative differences in the potential function.

\begin{tabular}{lllll}
\hline \hline Rotation symmetry & Time dependence & Mean velocity & Second moments & Vertex deviation \\
\hline & & Axial symmetry & & \\
$\frac{\partial}{\partial \theta}=0$ & $\frac{\partial}{\partial t}=0$ & $\Theta_{0}$ & $\mu_{\varpi \varpi}, \mu_{\theta \theta}, \mu_{z z}, \mu_{\varpi z}$ & $\varepsilon=0$ \\
$\frac{\partial}{\partial \theta}=0$ & $\frac{\partial}{\partial t} \neq 0$ & $\Pi_{0}, \Theta_{0}, Z_{0}$ & $\mu_{\varpi \varpi}, \mu_{\theta \theta}, \mu_{z z}, \mu_{\varpi z}$ & $\varepsilon=0$ \\
\hline & Point-axial symmetry & & \\
$f(\theta)=f(\theta+\pi)$ & $\frac{\partial}{\partial t}=0$ & $\Pi_{0}, \Theta_{0}, Z_{0}$ & $\mu_{\varpi \varpi}, \mu_{\theta \theta}, \mu_{z z}$, & $\varepsilon=\varepsilon(\theta)$ \\
& & & $\mu_{\varpi \tau}, \mu_{\varpi \theta}, \mu_{\theta z}$ & \\
$f(\theta)=f(\theta+\pi)$ & $\frac{\partial}{\partial t} \neq 0$ & $\Pi_{0}, \Theta_{0}, Z_{0}$ & $\mu_{\varpi \varpi}, \mu_{\theta \theta}, \mu_{z z}$, & $\varepsilon=\varepsilon(\theta)$ \\
& & & $\mu_{\varpi z}, \mu_{\varpi \theta}, \mu_{\theta z}$ & \\
\hline
\end{tabular}

six-dimensional phase space defined by star's position and velocity, can be assumed of normal type in the peculiar velocities,

$$
f(t, \boldsymbol{r}, \boldsymbol{V}) \propto \mathrm{e}^{-\frac{1}{2}(Q+\sigma)}
$$

where $Q$ is a positive definite quadratic form. Let $\boldsymbol{v}$ be the mean velocity, then $Q \equiv(\boldsymbol{V}-\boldsymbol{v})^{T} \cdot \boldsymbol{A} \cdot(\boldsymbol{V}-\boldsymbol{v})=1$ is the velocity ellipsoid, $\boldsymbol{A}(t, \boldsymbol{r})$ is a symmetric second-rank tensor, and $\sigma(t, \boldsymbol{r})$ a scalar function, both depending on time and position. Though $f$ could be assumed an arbitrary quadratic function of the integrals of motion of the stars, the Gaussian hypothesis represents an agreement point between gas dynamics theory, algebraic simplicity, the central limit theorem, and the bayesian criterion of less prior information.

The dynamic of such a kinematic component is then locally described according to a conservative dynamic system. The stars move under a common gravitating potential, and their phase space distribution function satisfies the Liouville equation. The substitution of Eq. (1) into Liouville equation leads to the well known Chandrasekhar system of equations (Chandrasekhar 1942). Since it is a linear differential system in $f$, the superposition principle can be applied in order to obtain the kinematic parameters of each stellar population, as well as the shape of the potential function.

It is also useful to characterize the geometry of the stellar system in order to assume specific symmetries that simplify the algebraic development and solution of system equations. The most usual simplified hypotheses are:

- Spatial symmetries: symmetry plane, axisymmetry and point-axial symmetry of the stellar system.

- Time dependence: stationary potential and steady state stellar system.

- Differential movement: non-differential movement of stellar components in a given direction.

A discussion on the consequences of assuming such symmetries, in the context of the velocity distribution, can be found in Paper I and in Alcobé \& Cubarsi (2004). Table 1 shows a summary of relevant kinematic parameters (in galactocentric cylindrical coordinates) depending on the symmetry or time dependence that is assumed in the model. We point out a few aspects addressed to the discussion of our results. Chandrasekhar had demonstrated that a steady-state stellar system must have an axisymmetric potential function. A generalization of Chandrasekhar systems was carried out by Orús (1952). Axisymmetric systems were studied by Català-Poch (1972), Sala (1990) and, under superposition, by Cubarsi (1990). However, under quite general potential functions, axisymmetric models were not able to explain the vertex deviation of velocity ellipsoids and a non-vanishing radial mean velocity. If axisymmetry is replaced by the point-axial symmetry hypothesis, where $f(\theta)=f(\theta+\pi)$ for the phase space distribution and the potential functions, then it allows to explain possible non-cylindrical features, like bars, spiral arms, elliptical movement of centroids, angular dependence of the vertex deviation $\varepsilon(\theta)$, etc. (Sanz-Subirana \& Català-Poch 1987; Juan-Zornoza 1995). Therefore, the point-axial assumption, together with the superposition principle, in order to give rise to non-null odd-order moments, are able to explain the main kinematic and dynamic features of stellar systems. For example, most samples drawn from CNS3 (Gliese \& Jahreiß1991) and HIPPARCOS (ESA 1997) catalogues lead to some non-null moments related to the vertex deviation, as well as non-null third moments (e.g. Paper I).

For point-axial systems, a measure of deviation from axial hypothesis is the axiallity index $I$ (Juan-Zornoza 1995) obtained from Oort's constants according to $I=C /(A+B)$. This quantity is defined from the second central moments, so that $I=-\mu_{\varpi \theta} / \mu_{\theta \theta}$. Similarly, the radial mean velocity depends on the index I. While for an axial system the radial mean velocity is obtained as $\Pi_{0}^{(\mathrm{cyl})}=\frac{1}{2} \frac{\dot{k}_{1}}{k_{1}} \varpi$, with $k_{1}$ an arbitrary function of time, for a point-axial system it depends on the axiallity index and on the mean rotation velocity $\Theta_{0}$, so that it can be written as follows,

$\Pi_{0}=\Pi_{0}^{(\mathrm{cyl})}-I \Theta_{0}$

Hence, above equation explains the deviation from circular motion of the centroid, since it predicts an elliptical movement 
whose radial direction is related to the vertex deviation moment $\mu_{\varpi \theta}$.

The outlined properties about symmetry hypotheses will provide a framework for the interpretation of single kinematic components after applying the segregation model.

\subsection{Mixture distribution}

Let $P$ be a parameter representing a property that delimits a stellar sample, for example, the distance to the Galactic plane for a fixed local cylinder, the modulus of the perpendicular velocity in a particular volume of the Galaxy, the absolute value of the star's velocity, etc. Requirements for such a parameter will be discussed in Sect. 2.3. Then, a subsample $S(P)$ may be drawn from an overall stellar sample, with a number of stars noted by \#S(P). We assume that a finite number of stellar populations, $c$, is present in $S(P)$, so that a partition of populations $A=\left\{A_{1}, A_{2}, \ldots, A_{c}\right\}$ composes the subsample.

For fixed time and position, the velocity density function, $f(\boldsymbol{V})$, is expressed as a mixture of $c$-partial density functions $\mathrm{g}^{(i)}\left(\boldsymbol{V} \mid \boldsymbol{C}^{(i)}\right)$, which are associated with stellar components depending on a set of population constants $\boldsymbol{C}^{(i)}$. It may be written as

$f(\boldsymbol{V})=\sum_{i=1}^{c} p\left(A_{i}\right) g^{(i)}\left(\boldsymbol{V} \mid \boldsymbol{C}^{(i)}\right)$

where $p\left(A_{i}\right)$ is the mixture proportion of the $i$ th population. Obviously $\sum_{i=1}^{c} p\left(A_{i}\right)=1$. The partial densities are assumed as normal trivariant functions for all the populations according to Eq. (1). Hence we can write $g^{(1)}=g^{(2)}=\ldots=g^{(c)} \equiv g$, and they depend on population parameters $\boldsymbol{C}^{(i)}=\left\{\boldsymbol{v}^{(i)}, \boldsymbol{M}_{2}^{(i)}\right\}$, where $\boldsymbol{v}^{(i)}$ is the mean velocity and $\boldsymbol{M}_{2}^{(i)}$ the second order central moment or covariance matrix of the $i$ th population.

We are interested in to stress the relationship between the mixture parameters and the selecting property $P$ of the subsample $S(P)$, hence we write $p\left(A_{i}\right)=n^{(i)}(P)$ and $\boldsymbol{C}^{(i)}=\boldsymbol{C}^{(i)}(P)$. Thus the density function of Eq. (3) becomes,

$f(\boldsymbol{V} \mid P)=\sum_{i=1}^{c} n^{(i)}(P) g\left(\boldsymbol{V} \mid \boldsymbol{C}^{(i)}(P)\right)$.

In order to estimate the mixture parameters we have applied the numerical-statistical method described in Paper I, although the foregoing concepts are quite general and independent of the segregation method. The velocity distribution is approximated from a two-component normal trivariate mixture, by using the total central moments and cumulants up to fourth order. A $\chi^{2}$ test is used to evaluate the error and the reliability of the approach.

In our original segregation algorithm, if more than two components were present in a sample, under certain conditions, the method separated the extreme and most dispersed population from the others, and the less dispersed populations were mixed up in a new component alone. Then, the algorithm could be recursively applied (Alcobé 2001).

The conditions enabling a recursive segregation were basically three: (1) the mean velocities of the mixture components had to preserve an order, for example increasing or decreasing in any of the velocity components; (2) consecutive population means had to be enough differentiated, as much as the velocity dispersion of the less dispersed population in the corresponding velocity component; (3) the population dispersions had to be non-decreasing in the direction inducing the hierarchy. Therefore, a recursive segregation did work from grater to smaller velocity samples. This facts suggested the idea of constructing a hierarchical set of samples by means of an ideal sampling parameter $P$, so that the recursive segregation does work from smaller to greater velocity samples, by improving the original procedure, and without needing of the third condition.

\subsection{Entropy of the mixture probability}

Let us review the relationship between probability and entropy (see e.g. Papoulis 1989). The function

$z(t)=\left\{\begin{array}{c}-t \ln t, 0<t \leq 1 \\ 0, t=0\end{array}\right.$

is non-negative, continuous and strictly concave. It allows to evaluate the entropy $H(A)$ of a partition $A=\left\{A_{1}, A_{2}, \ldots, A_{c}\right\}$, with probabilities $p\left(A_{i}\right)$, respectively, according to the following expression,

$H(A)=\sum_{i=1}^{c} z\left(p\left(A_{i}\right)\right)$

With the foregoing notation, for a sample $S(P)$, the entropy can be written explicitly depending on the parameter $P$, as follows

$H\left(A_{i} \mid P\right)=\sum_{i=1}^{c}-n^{(i)}(P) \ln \left(n^{(i)}(P)\right)$.

Above equation can be interpreted as the expected value of the uncertainty $I\left(A_{i} \mid P\right)=-\ln \left(n^{(i)}(P)\right)$, so that entropy variations measure uncertainty variations in the mixture parameters. The greater the entropy, the less the information of the population parameters. In general, most useful parameters are those describing large populations, instead of the ones describing an excessively informative partition, for example with very few stars in one group.

Hence, we are interested in to determine samples $S(P)$ that provide mixture parameters associated with maximum values of $H$. This will be done by tuning the parameter $P$. Indeed only a two-component mixture model is needed, since the entropybased algorithm will detect discontinuities of the entropy every time that one new population is merged to the sample.

In a two-component partition $\left\{A_{1}, A_{2}\right\}$, for a fixed $P$, taking $n \equiv n^{(1)}, n^{(2)}=1-n$, we can express the entropy as

$H(n(P))=-n(P) \ln (n(P))-(1-n(P)) \ln (1-n(P))$.

If we assume that the first population $A_{1}$ is the prominent one $\left(n \geq \frac{1}{2}\right), H$, as a function explicitly depending on $n$, is a positive, decreasing and differentiable function which satisfies

$0<H(n)<\ln 2$ and $\frac{\mathrm{d} H}{\mathrm{~d} n}<0, \quad$ for $\frac{1}{2}<n<1$. 


\subsection{Ideal sampling parameter}

An ideal sampling parameter should work in the following way. If a global sample is composed of a set of populations $\left\{A_{1}, A_{2}, \ldots, A_{c}\right\}$, a hierarchical set of subsamples $S(P)$ should progressively incorporate the populations $A_{1}, A_{2}$ and so on, by preserving an order, as continuously increasing the parameter $P$.

Then, for a two-component partition $\left\{A_{1}, A_{2}\right\}$ the sampling parameter $P$ should satisfy the following conditions:

C1 The number of stars of the subsample $S(P)$ increases with $P$, without losing any star. In other words, for two parameter values $P_{1}$ and $P_{2}$, we have

$$
P_{1}<P_{2} \Rightarrow S\left(P_{1}\right) \subseteq S\left(P_{2}\right) \Rightarrow \# S\left(P_{1}\right) \leq \# S\left(P_{2}\right) .
$$

C2 $P$ should induce an ordered incorporation of stars to the subsample $S(P)$ so that, as $P$ increases, stars of population $A_{1}$ are first included in the sample, and when such process has been completed, stars of population $A_{2}$ are successively merged to it. Hence, the mixture proportion of the first population satisfies

$P_{1}<P_{2} \Rightarrow n\left(P_{1}\right) \geq n\left(P_{2}\right)$.

More precisely, while the alone $A_{1}$-population increases, we have a constant population fraction

$n\left(P_{1}\right)=n\left(P_{2}\right)=1$

but, in presence of the incoming $A_{2}$-population, the following inequality is always fulfilled,

$n\left(P_{1}\right)>n\left(P_{2}\right)$.

C3 If we assume that the transition between populations is enough smooth, then $n(P)$ is a continuous and differentiable function of $P$. Thus Eq. (11) is equivalent to $\frac{\mathrm{d} n}{\mathrm{~d} P} \leq 0$, being $n(P)$ a non-increasing function.

Under previous assumptions, and taking into account Eq. (9), the entropy $H(n(P))$, or simply $H(P)$, is a nondecreasing function of $P$ in the interval $\frac{1}{2}<n<1$ :

$\frac{\mathrm{d} H}{\mathrm{~d} P}=\frac{\mathrm{d} H}{\mathrm{~d} n} \frac{\mathrm{d} n}{\mathrm{~d} P} \geq 0$.

Therefore, the entropy evolves, depending on the parameter $P$, according to the following property

$P_{1}<P_{2} \Rightarrow H\left(P_{1}\right) \leq H\left(P_{2}\right)$.

Thus, for a stellar sample composed of two populations, if the second population is merged to a subsample containing only the first population, according to the hierarchy that has been induced by the ideal sampling parameter, then the entropy of the mixture probability is a non-decreasing function of the sampling parameter.

\subsection{Sample with more than two populations}

Now we may ask about the behaviour of the function $H(P)$ for a sample with three populations $\left\{A_{1}, A_{2}, A_{3}\right\}$. Let us assume a continuous incorporation of stars to the subsample $S(P)$ by increasing $P$. Then, whether a small number of stars is added to the subsample $S(P)$, one of the following situations is produced:

(a) The new stars belong to population $A_{1}$. For an ideal sampling parameter this is only possible if the subsample $S(P)$ does not contain $A_{2}$ - and $A_{3}$-stars (Eq. (12)).

(b) The new stars belong to population $A_{2}$. Case corresponding to Eq. (13).

(c) The new stars belong to $A_{3}$-population. If they are enough different from $A_{2}$ stars, hence also from $A_{1}$-population, the two-component segregation model should to mix up the two closest populations $A_{1}$ and $A_{2}$ in the first component, and reserve the second ellipsoid for the population $A_{3}$. Therefore, a third condition is required:

C4 The partition $\left\{A_{1}, A_{2}, A_{3}\right\}$ is reduced to a two-component partition, with a cumulative population $S_{2}=A_{1} \cup A_{2}$, or even the less restricted condition $A_{1} \cup A_{2} \subset S_{2}$, and the new population $A_{3}$. In this case the relationship of Eq. (13) is no more fulfilled. Instead, the initial values $n=1$ and $H(P)=0$ are reset.

Notice that, though for two populations $A_{1}$ and $A_{2}$ the segregation algorithm is able to discriminate two velocity ellipsoids with similar means, the entering population $A_{3}$ is only recognized as a new one if it has an enough differentiated mean from the others.

Hence, in a general case of more than two components, the failure of condition $\mathbf{C 4}$ would indicate that the sampling parameter is not able to completely induce the hierarchy in the subsamples, and some populations could remain mixed.

The behaviour of population entropy while increasing the sampling parameter can be summarized in the following way. For a three-population sample there exist a couple of values $P_{A}$ and $P_{B}$ (that corresponds to sampling parameter values for which the first and the second populations, respectively, have been completely included in the subsample) satisfying:

(1) if $P<P_{A}$ then $H(P)=0$. The subsample is only composed of $A_{1}$ stars;

(2) if $P \in\left(P_{A}, P_{B}\right)$ then $H(P)>0$ and $\frac{d H}{d P}>0$. During the mixture of two populations $A_{1}$ and $A_{2}$ there is an interval with continuously increasing entropy;

(3) if $P \longrightarrow P_{B}$, then $H(P) \longrightarrow 0$ and $n(P) \longrightarrow 1$. An entropy drop takes place when the new $A_{3}$-population, enough differentiated from $A_{2}$-population, appears.

Above situation is easily generalized for any greater number of populations, so that the case (c) can be repeated for each new entering population. Then, the entropy $H(P)$ can show several relative maxima and subsequent drops. Also notice that in each iteration, the informative population is the one entering, which 
produces the discontinuity, while the previous ones become indistinguishable in a cumulative component.

Obviously, it does not exist a strictly ideal kinematic parameter. Usually the entropy does not fall straight down to zero when the second population is completed, but it does show a strong drop. Hence we must look for relative maximum levels of entropy, or intervals with a continuous increasing entropy, followed by a significant downward slope, that indicates that a new population has been merged to the subsample and that stars of the next incoming population are being detected. In this way, it is possible to know how many populations are contained in the sample. Hence, if $k$ is the number of entropy maxima, the number of stellar populations in the sample is $k+1$.

Let us remark that, by continuity conditions, as a few new stars of a population already in the sample are entering, the entropy level remains slowly increasing, and the mixture parameters do not significantly vary. In the interval where that situation occurs, since the segregation method provides a $\chi^{2}$ fitting error, it is profitable to choose the sample $S(P)$ giving the minimum $\chi^{2}$.

Some candidates to ideal sampling parameters, that can serve to discriminate between kinematic populations, are, for a particular volume of the Galaxy, the velocity component perpendicular to the Galactic plane $\left|V_{3}\right|$, the rotational component $V_{2}$, the modulus of the star's velocity $|\boldsymbol{V}|$, or for a particular section of the disk, the distance $z$ to the Galactic plane. We have found that a small correction on $|\boldsymbol{V}|$, the modulus of the velocity referred to the cumulative local standard of rest, has the best selecting behaviour, specially for large samples and for more than three populations. The later will be referred as re-centred velocity.

\subsection{Monte Carlo simulation}

The entropy variations and the behaviour of the segregation algorithm, depending on the sampling parameter, can be illustrated through a numerical simulation from synthetic samples. The samples were generated from pseudo-random algorithms (Press et al. 1992) to get trivariate normal data. The simulations have been used for testing the reliability of the results depending on the number of data vectors of the input samples. The algorithm was able to segregate normal population samples with around 100 data vectors, otherwise the normality of these components could not be asserted, for example whether non-null third central moments are obtained. A successful segregation was always reached if the difference between the means was greater, at least in one direction, than the standard deviation of the less dispersed population, although in some cases, depending on the other velocity components, this amount could be reduced to the half (Alcobé 2001). The data vectors for star velocities were originally obtained as three independent normal variates. Then the vectors were rotated around two coordinate axes in order to get a velocity ellipsoid with an arbitrary orientation, and hence trivariate vectors.

The Fig. 1 illustrates several typical situations for a fourcomponent mixture. In the first row of graphics, the first plot shows the stellar density $N(P)$ as a function of the sampling parameter. In this case example a superposition of four pure Gaussian populations have been assumed. The second plot shows the theoretical entropy behaviour when the segregation is made by using the prior information about the population the sampling parameter is scanning. The third plot shows the entropy curve when the segregation method works without any prior information. Thus, three relative entropy maxima are obtained according to a four-component mixture.

In the second row of graphics, the simulation of a real situation is shown. Now synthetic samples have been used, which have some random deviations from Gaussianity. Moreover, the mixture components have quite overlapping wings. Once again, when the segregation is carried out with the information about the population that is being scanned, only small waves of entropy are produced, due to the fluctuations of the entering population distribution. However, if the segregation algorithm works without any prior information, the overlapping wings produce a smaller entropy drop, but still very significant. In this example the small entropy oscillations are associated with fluctuations around $10 \%$ of the entering population, while local maxima still reflect the total number of stars involved in the bimodal pattern.

\subsection{Naming the method}

The combination of the sample filtering procedure and the segregation method provides an efficient algorithm which allows to determine the kinematic parameters (i.e. mean velocities, dispersions and mixture proportions) of the populations composing the stellar sample. For a hierarchical set of subsamples, each relative maximum level of the entropy of the mixture probability provides an optimal segregation containing the last entered population, while the fitting error of the segregation method allows to assert whether the populations are Gaussian. The method will be referred as MEMPHIS, Maximum Entropy of the Mixture Probability from HIerarchical Segregation.

\section{Stellar samples}

\subsection{HIPPARCOS samples}

In order to carry out our analysis, a stellar sample composed of a large number of stars with known velocity space was selected. It was obtained by crossing HIPPARCOS Catalogue with radial velocities from Hipparcos Input Catalogue HINCA, with 19466 stars (see Paper I). The completeness of the catalogue is reduced for larger distances since it has been limited by star magnitude, and the sample was limited to a trigonometric distance of $300 \mathrm{pc}$. The resulting sample was composed by 13678 stars, where the only input data were the velocity components $\left(V_{1}, V_{2}, V_{3}\right)$ in a cartesian heliocentric coordinate system, with $V_{1}$ toward the direction of Galactic centre, $V_{2}$ in the rotational direction, and $V_{3}$ perpendicular to Galactic plane, positive in the direction of the North Galactic pole.

It is well known that stellar samples with radial velocities are biased toward stars with high-proper motions (Binney et al. 1997). Nevertheless, in Paper I we had exhaustively analyzed the features of our working sample, where we found a 

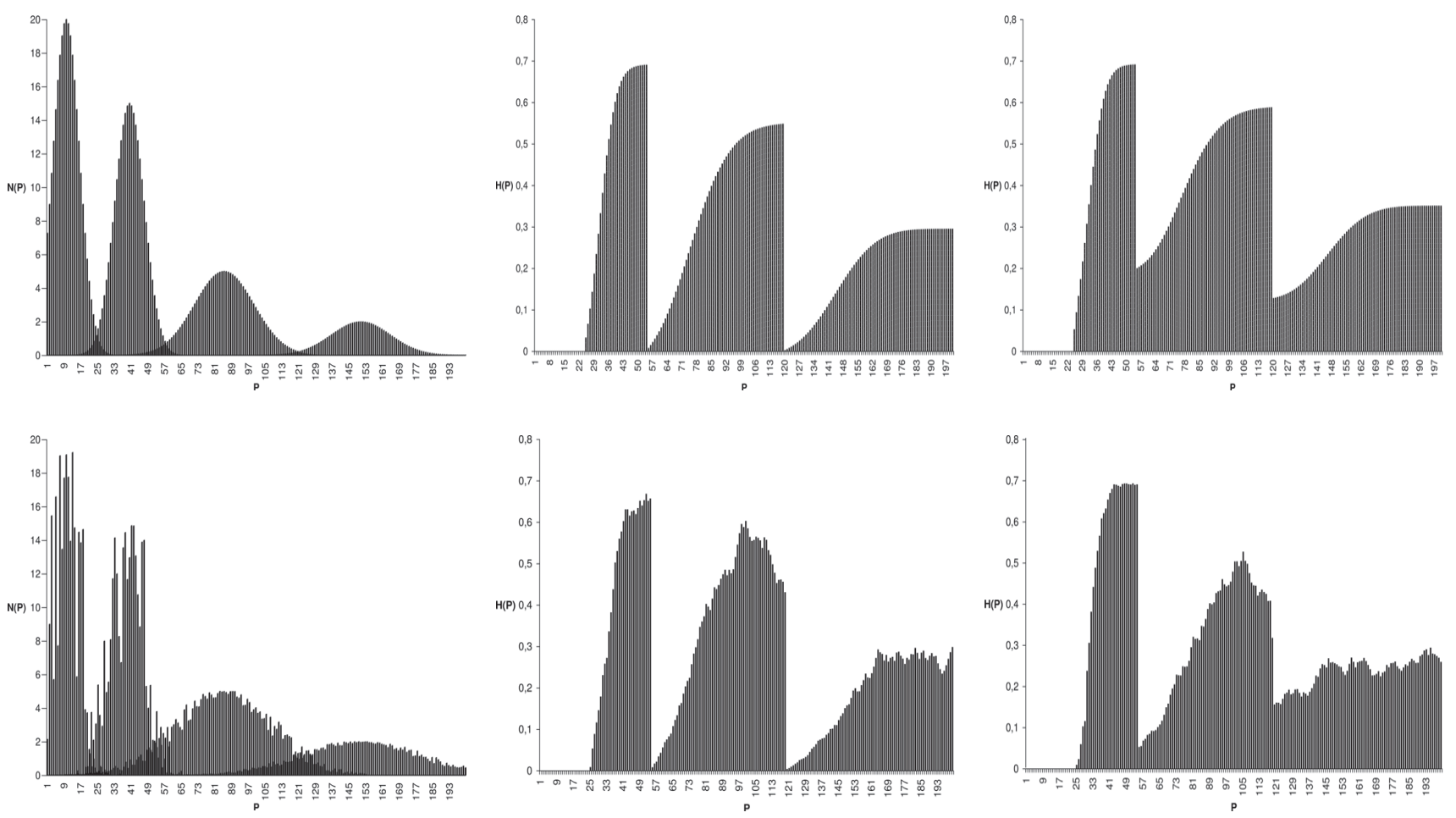

Fig. 1. First row: the first plot shows the stellar density $N(P)$ as a function of the sampling parameter for a simulated case example. A superposition of four pure Gaussian populations was assumed. The second plot shows the entropy variations the exactly known mixture. The third plot shows the entropy curve when the segregation method works with no prior information. The four-component mixture provides three net local maxima. second row: the synthetic populations have been used, which have some random deviations from Gaussianity and a significant superposition of partial distribution wings. The resulting segregation, working from the prior information about the real population components (second plot) produces small entropy waves (here, the small entropy oscillations are associated with fluctuations around $10 \%$ of the new entering population), but if the segregation method works without such information (third plot) then the effect of the overlapping wings produces a smaller but still significant entropy drop.

homogeneous and unbiased kinematic behaviour within each mixture component.

\subsection{Application of MEMPHIS}

In Paper I the sample was selected by applying the criterion of taking the modulus of its mean velocity, $v$, and their total standard deviation, $\sigma_{v}$, so that the star velocities satisfy $|\boldsymbol{V}|<v+k \sigma_{v}$, with $k$ between 2 and 3. Then, among the foregoing samples, we looked for the best approximation of the velocity distribution by a two-component mixture, given by the minimum $\chi^{2}$ error. Now, the selection of the subsamples will be done according to Sect. 2.4. The subsamples are obtained looking for maxima of entropy of the mixture probability. However if $|\boldsymbol{V}|$ is chosen as sampling parameter, with the velocity referred to the Sun, then a bias could be introduced due to the eventual exclusion of stars from the sample whose heliocentric velocity modulus is greater than the imposed limit, but closer to the mean of the population where they belong to. In order to avoid this bias, we shall apply the selection procedure in two steps.

1) Predictor step: MEMPHIS is applied to the heliocentric subsample, filtered by the sampling parameter corresponding to the last maximum of entropy.
2) Corrector step: the star velocities are displaced to the cumulative subcentroid, that is the mean of the cumulative subsample corresponding to each entropy maximum, and MEMPHIS is applied again by selecting stars with the modulus of the velocity referred to the new origin.

Obviously, this iterative procedure is irrelevant in a twocomponent mixture, but it leads to a significant improvement for three or more populations. Thus the corrected sampling parameter becomes more ideal and it improves the contrast between entropy drops and maxima.

\subsection{Heliocentric subsamples}

After scanning the whole sample, two main relative high levels of entropy are found. As main we mean either a relative maximum of entropy followed by a significant entropy drop, or a constant level of entropy before a decreasing interval. As expected, some oscillations around the main maxima are detected. Such variations can be interpreted as produced by small groups of stars, whose characteristic parameters are slightly different from those of the population where they belong to. They could be stars from moving groups. Furthermore, around a relative maximum of entropy, the relative minimum of $\chi^{2}$ provides us with the best fit. Anyway, the resulting central moments of the distribution are quite stable for small variations 


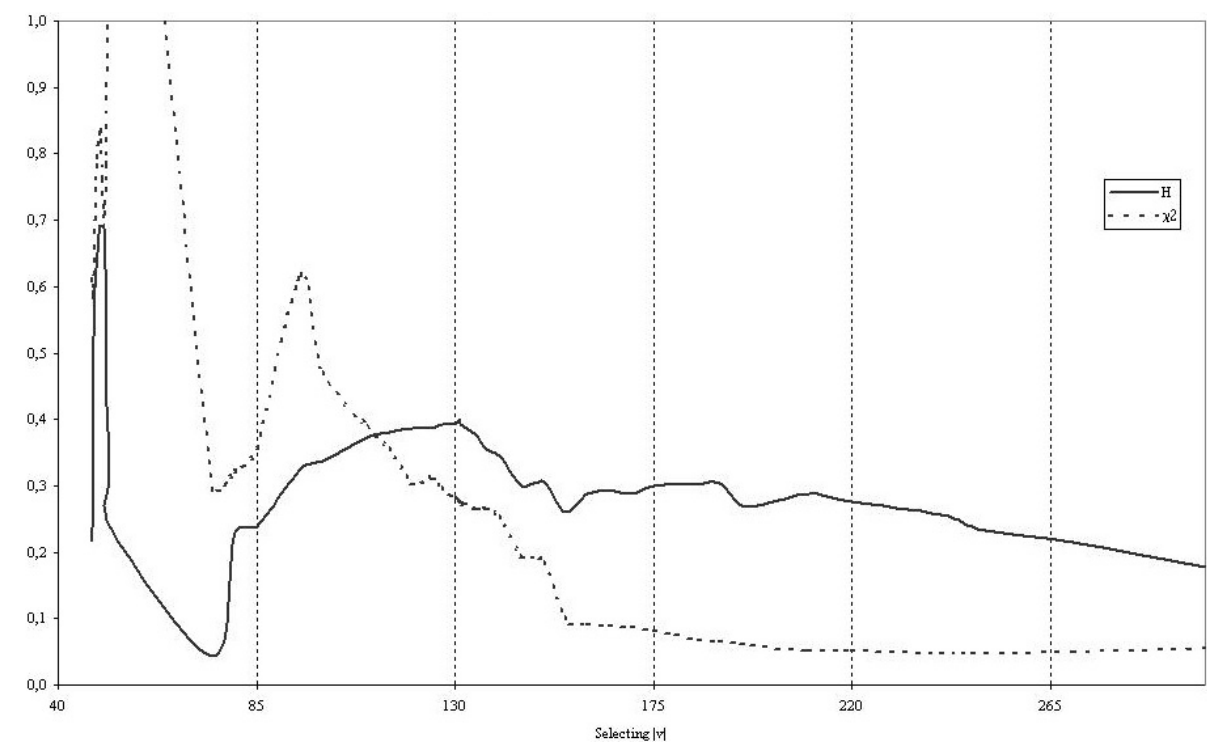

Fig. 2. Entropy variations for HIPPARCOS heliocentric samples are plotted as continuous line depending on the selecting maximum velocity of the sample $|\boldsymbol{V}|\left(\mathrm{km} \mathrm{s}^{-1}\right)$. Relative variations of $\chi^{2}$ are also showed (dashed line).

Table 2. HIPPARCOS heliocentric subsamples: Mixtures at maximum population entropy, predictor step. The sampling parameter is $P=|\boldsymbol{V}|_{\max }$ referred to the Sun.

\begin{tabular}{ccccrrrrrrrr}
\hline \hline$P$ & $\chi^{2}$ & $\# S(P)$ & Pop. & $\mu_{11}$ & $\mu_{22}$ & $\mu_{33}$ & $\mu_{12}$ & $v_{1}$ & $v_{2}$ & $v_{3}$ & $n$ \\
\hline 51 & \multirow{2}{*}{150} & \multirow{2}{*}{10195} & $A_{1}$ & 135 & 116 & 44 & 3.3 & -21.3 & -14.7 & -5.9 & 0.50 \\
& & & $A_{2}$ & 251 & 208 & 189 & -1.6 & 6.3 & -7.7 & -6.9 & 0.50 \\
\hline 131 & \multirow{2}{*}{135} & \multirow{2}{*}{515} & $S_{2}$ & 742 & 254 & 167 & 82 & -10.4 & -13.5 & -7.2 & 0.90 \\
& & & $A_{3}$ & 2722 & 843 & 879 & 280 & -9.7 & -48.1 & -7.2 & 0.10 \\
\hline 209 & \multirow{2}{*}{12} & \multirow{2}{*}{13530} & $S_{3}$ & 794 & 258 & 160 & 93 & -10.7 & -14.1 & -7.2 & 0.91 \\
& & & $A_{4}$ & 4275 & 1371 & 1642 & 333 & -13.0 & -63.1 & -7.9 & 0.09 \\
\hline
\end{tabular}

of entropy. Notice that, if both population fractions are similar $(n=0.5)$, the entropy is the highest possible $(H(0.5)=\ln 2)$, hence the drops are more visible, but if the first component of the mixture becomes dominant, the population entropy is getting much lower $(H(1)=0)$, and ulterior drops are smaller. Figure 2 shows the entropy evolution depending on the sampling parameter $|\boldsymbol{V}|$.

The main relative maxima of entropy are placed at $P_{1}=51$ and $P_{2}=131 \mathrm{~km} \mathrm{~s}^{-1}$. Also, there is a large interval, from 155 to $220 \mathrm{~km} \mathrm{~s}^{-1}$, where the entropy remains nearly constant, with a small relative maximum at $P_{3}=209 \mathrm{~km} \mathrm{~s}^{-1}$ and a relative minimum of $\chi^{2}$. Tables 7-9 show the velocity moments and cumulants up to fourth order for the subsamples selected according to the foregoing values of the sampling parameter.

Table 2 shows the mixture parameters for pairs of segregated components, where $P=|\boldsymbol{V}|_{\max }$ is the sampling parameter value, $\chi^{2}$ is the fitting error with 15 degrees of freedom, $\# S(P)$ is the number of stars of the subsample, Pop. names the population, $\mu_{i j}$ are the second central moments, $v_{i}$ is the mean heliocentric velocity, and $n$ is the mixture proportion. For the first entropy maximum, the large value of $\chi^{2}$ shows that the Gaussian hypothesis is not fulfilled. For the second maximum, the normal distribution of components is still inadequate but errors in the determination of kinematic parameters are much lower. For the third maximum, instead, the approximation by normal distributions becomes plausible. Though first and second maxima of entropy lead to not-Gaussian components, MEMPHIS has shown that important discontinuities in the velocity distribution have occurred at the corresponding values of $P$.

\subsection{Re-centred samples}

The catalogue is now displaced to the new velocity origin, corresponding to each one of the cumulative subcentroids (represented by $S_{i}$ in Table 2). Figure 3 shows the entropy evolution depending on the new sampling parameter $P$. Mixture parameters for pairs of components, which approximate each selected subsample, are shown in Table 3. Now the entropy maxima of the heliocentric sample are more stable.

The new representative entropy maxima are placed at $P_{2}=$ 124 and $P_{3}=209 \mathrm{~km} \mathrm{~s}^{-1}$. From $\chi^{2}$ values, a significant improvement of contrast is noticed, that leads to better approximations.

As we had commented, the significance of the relative maxima and the minor waves of entropy is related to the number of stars of the new detected population. After a local entropy maximum, the number of stars of the entering population suffers a worthy drop (see Fig. 4), while along the small oscillations it is much smaller. Also, as we shall discuss later, the moments of the first cumulative population remain nearly constant, while the kinematic parameters of the second population 


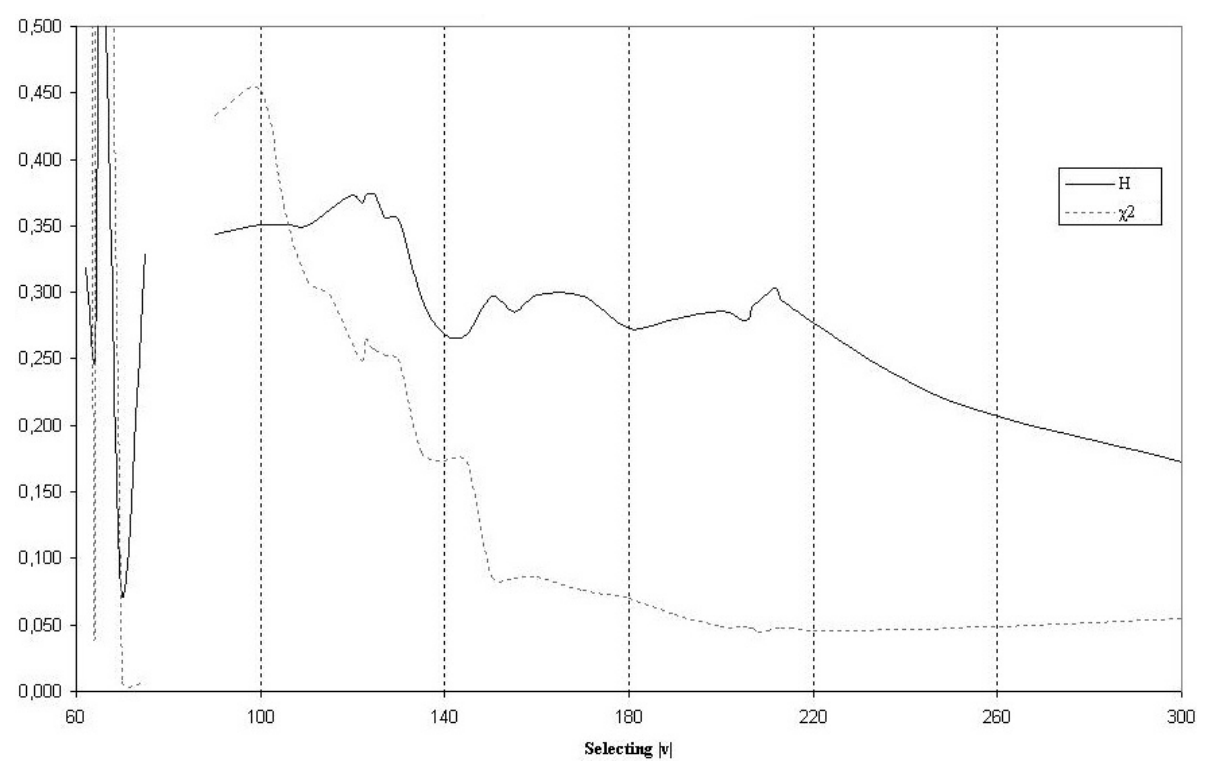

Fig. 3. Entropy variations for HIPPARCOS re-centred samples are plotted as continuous line depending on the selecting maximum velocity of the sample $|\boldsymbol{V}|\left(\mathrm{km} \mathrm{s}^{-1}\right)$. Relative variations of $\chi^{2}$ are also showed (dashed line).

Table 3. HIPPARCOS re-centred subsamples: Central second moments, mean velocities, and population fraction for mixtures at maximum population entropy. The sampling parameter is $P=|\boldsymbol{V}|_{\max }$ referred to the cumulative subcentroid.

\begin{tabular}{rcccrrrrrrrrrr}
\hline \hline$P$ & $\chi^{2}$ & $\# S(P)$ & Pop. & $\mu_{11}$ & $\mu_{22}$ & $\mu_{33}$ & $\mu_{12}$ & $\mu_{13}$ & $\mu_{23}$ & $v_{1}$ & $v_{2}$ & $v_{3}$ & $n$ \\
\hline 51 & $>150$ & 10195 & $A_{1}$ & 135 & 116 & 44 & 3 & 55 & -17 & -21.3 & -14.7 & -5.9 & 0.50 \\
& & & & \pm 719 & 30 & 12 & 127 & 57 & 8 & 4.3 & 0.3 & 0.2 & 0.01 \\
& & & $A_{2}$ & 251 & 208 & 189 & -2 & -46 & 16 & 6.3 & -7.7 & -6.9 & 0.50 \\
& & & & \pm 710 & 30 & 11 & 125 & 57 & 8 & 4.3 & 0.3 & 0.2 & 0.01 \\
\hline 124 & 75 & 13325 & $S_{2}$ & 730 & 248 & 162 & 81 & -7 & 5 & -10.3 & -13.3 & -7.2 & 0.89 \\
& & & \pm 21 & 30 & 9 & 9 & 8 & 5 & 0.3 & 0.2 & 0.1 & 0.01 \\
& & & $A_{3}$ & 2624 & 853 & 866 & 265 & -65 & 84 & -11.6 & -47.8 & -7.2 & 0.11 \\
& & & \pm 116 & 231 & 54 & 43 & 46 & 28 & 0.4 & 1.0 & 0.1 & 0.01 \\
\hline 209 & 12 & 13541 & $S_{3}$ & 796 & 249 & 157 & 91 & -4 & 4 & -10.6 & -14.0 & -7.2 & 0.91 \\
& & & \pm 33 & 69 & 16 & 15 & 12 & 10 & 0.4 & 0.3 & 0.3 & 0.01 \\
& & \multirow{2}{*}{$A_{4}$} & 4288 & 1525 & 1710 & 334 & -150 & 66 & -14.7 & -64.8 & -8.3 & 0.09 \\
& & & \pm 280 & 710 & 149 & 117 & 99 & 79 & 2.9 & 2.7 & 2.7 & 0.01 \\
\hline
\end{tabular}

sensibly vary. The velocity moments of the entering population become more significant as larger is this population. For this reason, the local maximum of entropy, the relative maximum of second population stars, and the relative minimum of $\chi^{2}$ are nearly simultaneous.

\subsection{Total number of components}

According to its definition, the sampling parameter introduces a hierarchy in the subsamples so that the smaller $P$, the smaller number of populations contained in the subsample. As explained in case (c) of Sect. 2.4, if the value $P_{1}$ of the sampling parameter leads to the segregation of populations $A_{1}$ and $A_{2}$, the next value $P_{2}$ builds a partition with $S_{2} \approx A_{1} \cup A_{2}$ and $A_{3}$. In general, the $i$ th maximum of entropy, defined by the parameter $P_{i}(i>1)$, leads to a partition formed by the cumulative population $S_{i} \approx \cup_{k=1}^{i} A_{k}$, and the last entered population $A_{i+1}$. Therefore, as we have got three relative maxima of entropy, we should have four different populations in the total sample. Let us note that there is a difference of more than $10 \%$ stars between the subsample corresponding to the first maximum of entropy, $A_{1} \cup A_{2}$, and the cumulative population $S_{2}$ of the second maximum. This means, $\# S_{2} \neq \# A_{1}+\# A_{2}$, but $\# S_{2}=\# A_{1}+\# A_{2}+\# B_{2}$, with $\# B_{2}=1,485$ stars. Thus, $S_{2}$ is composed of two discrete subcomponents, $A_{1}$ and $A_{2}$, and other stars, $B_{2}$ which are mixed with the above subcomponents within $S_{2}$.

What happens with $A_{3}$ is more interesting. The segregation induced by the parameter $P_{2}=124$ leads, on one hand, to a population $S_{2}$ (11726 stars) with a similar kinematics to $S_{3}$ (12326 stars) obtained from $P_{3}=209$, and, on the other hand, to a population $A_{3}$ (1465 stars), which is kinematically distinguishable from $S_{2}$, for example by looking at the shape of the velocity ellipsoids. Then, the population $A_{3}$ is broken into two groups $B_{3}$ (about 465 stars) and $C_{3}$ (about 1000 stars), so that $B_{3}$ is merged to $S_{2}$, by giving $S_{3}$, without modifying in a 


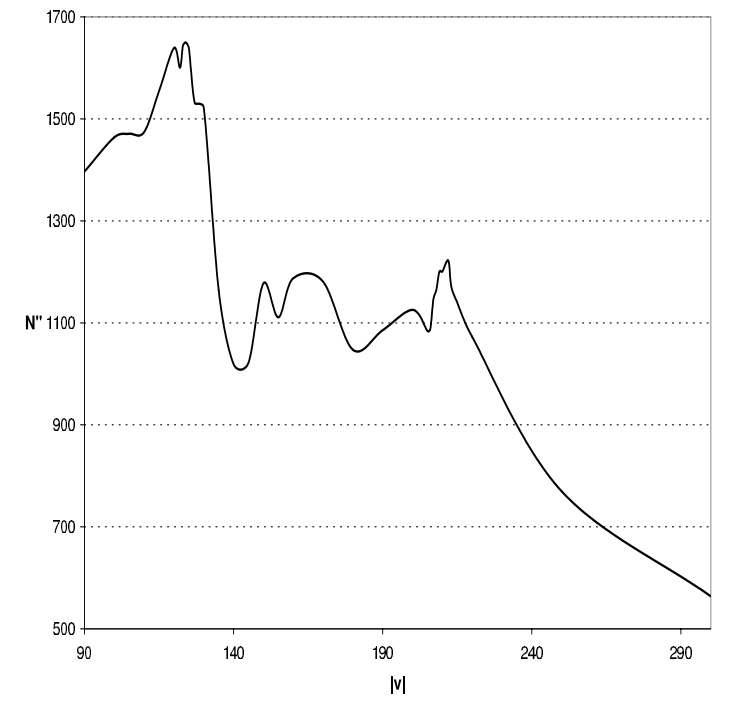

Fig. 4. The number of stars that are identified as the new entering population, $N^{\prime \prime}$, is plotted as a function of the sampling parameter. The entropy drop around $|\boldsymbol{V}|=130 \mathrm{~km} \mathrm{~s}^{-1}$ is highly significant, since an amount of 650 stars are involved, which represent about $40 \%$ of that population. On the contrary, the small oscillations from 140 to $220 \mathrm{~km} \mathrm{~s}^{-1}$ only represent relative variations lesser than $18 \%$. These fluctuant stars belong to the region where the wings of the partial distributions are overlapping, and the segregation method interprets them alternatively either from the first or from the second population, up to reach the optimal parameter value.

significant way the shape of the velocity ellipsoid, and $C_{3}$ is included within $A_{4}$, which is a stellar component normally distributed. Let us note that the increase in moment $\mu_{11}$ from the subsample with $A_{2}$ (Table 7 ) to the sample $S_{2}$ is due to a group of stars with a radial dispersion similar to the one of $A_{3}$. Since $\# S_{3}<\# S_{2}+\# A_{3}$, the condition $\mathbf{C 4}$ of Sect. 2.4 is not fulfilled. Thus, $B_{3}$ seems to be a broad wing of $S_{2}$, while $C_{3}$ belongs to the wing of $A_{4}$, which are overlapping. Such situation will be further analyzed in following sections. Therefore, although the sampling parameter is unable to completely unfold this group of stars, we interpret the entropy level around $P=124$ as associated with the detection of a new population.

Therefore, we have found the non-Gaussian subcomponents $A_{1}, A_{2}$ and $A_{3}$, and the Gaussian components $S_{3}$ and $A_{4}$.

\subsection{Results}

The final kinematic parameters for the three segregations of Table 3, expressed in terms of standard deviations and vertex deviation, $\varepsilon$, are the following ones:

$A_{1}$ subcomponent with $\left(\sigma_{1}, \sigma_{2}, \sigma_{3}\right)=(12 \pm 31,11 \pm 1,7 \pm 1)$. $\mathrm{A}_{2}$ subcomponent with $\left(\sigma_{1}, \sigma_{2}, \sigma_{3}\right)=(16 \pm 22,14 \pm 1,14 \pm 1)$. $S_{3}$ Gaussian component with $\left(\sigma_{1}, \sigma_{2}, \sigma_{3}\right)=(28 \pm 1,16 \pm$ $2,13 \pm 1), \varepsilon=10 \pm 2^{\circ}$.

$A_{3}$ subcomponent with $\left(\sigma_{1}, \sigma_{2}, \sigma_{3}\right)=(51 \pm 1,29 \pm 4,29 \pm 1)$, $\varepsilon=8 \pm 2^{\circ}$.

$A_{4}$ Gaussian component with $\left(\sigma_{1}, \sigma_{2}, \sigma_{3}\right)=(65 \pm 2,39 \pm$ $9,41 \pm 2), \varepsilon=7 \pm 3^{\circ}$.

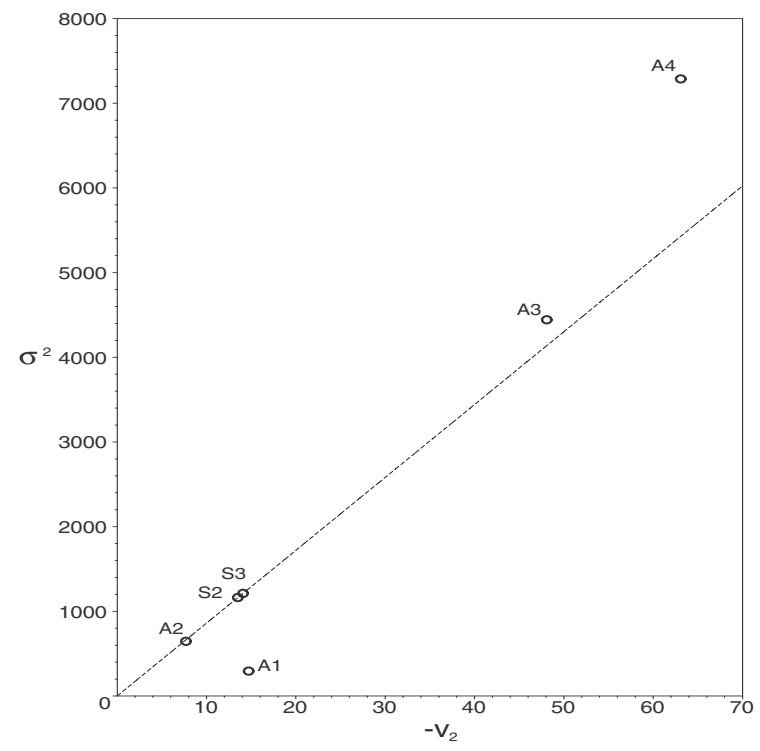

Fig. 5. For the stellar populations obtained from HIPPARCOS subsamples, the total velocity dispersion $\sigma^{2}$ is plotted versus the opposite of the mean heliocentric rotation velocity. The fitting of young disk stars, $A_{2}$, and two thin disk evaluations, $S_{2}$ and $S_{3}$, containing an increasing number of old disk stars allowed us to estimate the slope of Strömberg's law for the asymmetric drift of disk stars, $\rho=-\sigma^{2} / v_{2}=86 \pm 3 \mathrm{~km} \mathrm{~s}^{-1}$. The population of early-type stars, $A_{1}$, as well as the increasing thick disk, from $A_{3}$ to $A_{4}$, do not fit in the linear relationship.

These groups may be interpreted as follows. If we begin with the Gaussian components, the third sampling parameter $P_{3}=$ 209 of Table 3 shows the best value of $\chi^{2}$. This cut excludes only few stars from the overall sample that can be considered noisy, with large velocity errors. For such sample, the obtained segregation is totally consistent with Gaussian distributions, and can be identified, as in Paper I, with thin disk, $S_{2} \approx S_{3}$, and thick disk, $A_{4}$.

The subcomponent $A_{1}$ shows velocity dispersions similar to early-type stars (Skuljan et al. 1999), it may be a stellar population belonging to a spiral arm (Dehnen \& Binney 1998). Such population has not a Gaussian distribution, especially in the radial direction, and their kinematic parameters are computed with large errors.

The subcomponent $A_{2}$ has a typical velocity dispersions of young disk population (see e.g. estimations of kinematic parameters for such components from the Besançon model in Ojha et al. 1994). Not either it has a Gaussian distribution, mainly in the radial velocity, and the given kinematic parameters are also orientative. Nevertheless we must point out that the asymmetric drift given by the ratio dispersion over heliocentric velocity is similar to the one of the thin disk, $\rho=-\sigma^{2} / v_{2}=$ $86 \pm 3 \mathrm{~km} \mathrm{~s}^{-1}$ (Fig. 5).

The subcomponent $A_{3}$ has a wider velocity ellipsoid with a ratio $\sigma_{2} / \sigma_{3} \approx 1$, similarly to the thick disk. The asymmetric drift relation, $\rho=91 \pm 6$, is slightly different from the one of the thin disk. The whole thick disk is $\rho=116 \pm 13$. Thus, we could identify the subcomponent $A_{3}$ as the beginning of the thick disk $A_{4}$, although still mixed with old thin disk stars. Therefore, old 
Table 4. CNS3 heliocentric subsamples: Mixtures at maximum population entropy, predictor step. The sampling parameter is $P=|\boldsymbol{V}|_{\max }$ referred to the Sun.

\begin{tabular}{cccccrrrrrrr}
\hline \hline$P$ & $\chi^{2}$ & $\# S(P)$ & Pop. & $\mu_{11}$ & $\mu_{22}$ & $\mu_{33}$ & $\mu_{12}$ & $v_{1}$ & $v_{2}$ & $v_{3}$ & $n$ \\
\hline 40 & 114 & \multirow{2}{*}{897} & $A_{1}$ & 227 & 35 & 33 & -71 & -13.8 & -19.6 & 2.1 & 0.44 \\
& & & $A_{2}$ & 250 & 80 & 121 & -20 & 2.7 & -1.0 & -11.7 & 0.56 \\
\hline 135 & 29 & \multirow{2}{*}{1874} & $S_{2}$ & 1089 & 352 & 309 & 96 & -7.3 & -15.6 & -7.4 & 0.84 \\
& & & $A_{3}$ & 2389 & 1136 & 848 & -131 & -27.4 & -47.0 & -10.1 & 0.16 \\
\hline 180 & 23 & \multirow{2}{*}{1915} & $S_{3}$ & 1272 & 463 & 340 & 132 & 10.1 & -18.7 & -7.9 & 0.93 \\
& & & $A_{4}$ & 4759 & 2223 & 1895 & -62 & -29.8 & -63.9 & -7.5 & 0.07 \\
\hline
\end{tabular}

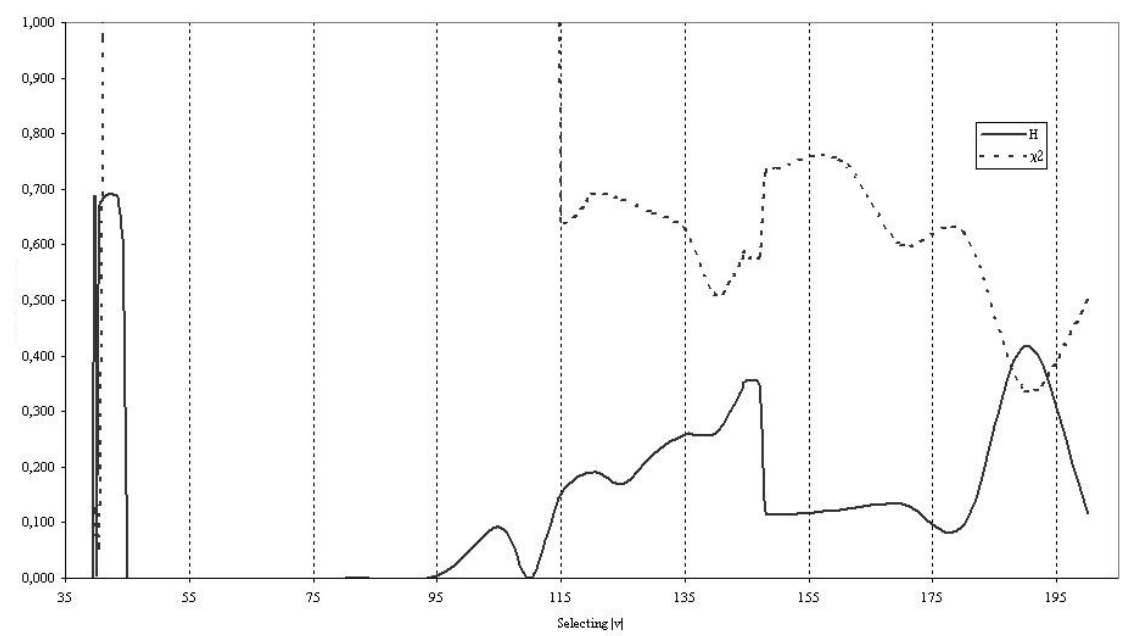

Fig. 6. Entropy variations for CNS3 re-centred samples are plotted as continuous line depending on the selecting maximum velocity of the sample $|\boldsymbol{V}|\left(\mathrm{km} \mathrm{s}^{-1}\right)$. Relative variations of $\chi^{2}$ are also showed (dashed line).

disk stars can be described as a continuous population composing $S_{3}-\left(A_{1} \cup A_{2}\right)$.

\subsection{CNS3 samples}

It is well known that HIPPARCOS is nowadays the most accurate astrometric catalogue, and results derived from it are the most reliable. Nevertheless, in order to compare our kinematic results, MEMPHIS has been also applied to a completely different catalogue. Such results may be also compared with similar ones obtained in earlier works (Cubarsi 1992; Alcobé \& Cubarsi 2001). The Third Catalogue of Nearby Stars includes all known stars within a distance of 25 pc from the Sun. Even though a lot of stars of CNS3 are present in HIPPARCOS Catalogue, there are also many others out of the limiting magnitude of HIPPARCOS satellite. So, the selected criteria for building CNS3 are not correlated with those used for defining HIPPARCOS. The sample has been obtained taking into account all stars from CNS3 with known velocity space. These are 1946 stars in total. As the subdwarfs are not considered belonging to the Galactic disk (Erickson 1975), six stars among them which are so described have been also rejected. Anyway, the obtained results are not significantly different to those derived if such stars were included. Nevertheless, as errors were slightly smaller if these stars are excluded, the criterion was maintained.
For CNS3 subsamples, similar maxima of entropy were found. These maxima for the heliocentric sample are placed on (see Table 4) $P_{1}=40, P_{2}=135$, and $P_{3}=180 \mathrm{~km} \mathrm{~s}^{-1}$. Tables 10-12 show their velocity moments and cumulants up to fourth order.

By repeating the process as for HIPPARCOS sample, the star velocities were displaced to the cumulative subcentroids computed from the heliocentric sample (Table 4).

Figure 6 shows the equivalent maxima for the re-centred samples. They show a shape very similar to ones from HIPPARCOS catalogue: The new maxima are placed at $P_{2}=$ 145 and $P_{3}=190 \mathrm{~km} \mathrm{~s}^{-1}$.

The first relative maximum leads to a segregation with large $\chi^{2}$ leading to a non Gaussian mixture. The second value of the sampling parameter, $P_{2}=145$, leads to a nearly Gaussian mixture, and the segregation from $P_{3}=190$ produces a clearly normal mixture.

Notice that the sampling parameter from heliocentric velocities (Table 4) leads to an intermediate population $A_{3}$. However it is totally deconvolved while working from re-centred peculiar velocities (Table 5), since the second and third segregations are nearly the same one (almost with the same number of stars).

Therefore, CNS3 sample is segregated into four components or subcomponents: two main stellar components with Gaussian distribution, and two stellar subcomponents. Their moments are shown in Table 5. 
Table 5. CNS3 re-centred subsamples: Central second moments, mean velocities, and population fraction for mixtures at maximum population entropy. The sampling parameter is $P=|\boldsymbol{V}|_{\max }$ referred to the cumulative subcentroid.

\begin{tabular}{ccccrrrrrrrrrr}
\hline \hline$P$ & $\chi^{2}$ & $\# S(P)$ & Pop. & $\mu_{11}$ & $\mu_{22}$ & $\mu_{33}$ & $\mu_{12}$ & $\mu_{13}$ & $\mu_{23}$ & $v_{1}$ & $v_{2}$ & $v_{3}$ & $n$ \\
\hline 40 & 114 & 897 & $A_{1}$ & 227 & 35 & 33 & -71 & 74 & 56 & -13.8 & -19.6 & 2.1 & 0.44 \\
& & & & \pm 196 & 192 & 137 & 171 & 163 & 143 & 3.6 & 1.9 & 4.4 & 0.02 \\
& & & $A_{2}$ & 250 & 80 & 121 & -20 & 42 & 76 & 2.7 & -1.0 & -11.7 & 0.56 \\
& & & & \pm 155 & 152 & 109 & 136 & 129 & 113 & 4.6 & 2.4 & 5.6 & 0.02 \\
\hline 145 & 26 & \multirow{2}{*}{1904} & $S_{2}$ & 1272 & 450 & 334 & 125 & -57 & 3 & -10.8 & -18.5 & -7.7 & 0.93 \\
& & & \pm 76 & 117 & 37 & 37 & 34 & 22 & 0.9 & 0.8 & 3.8 & 0.02 \\
& & \multirow{2}{*}{$A_{3}$} & 4240 & 1951 & 1840 & 556 & 564 & -145 & -18.3 & -63.8 & -7.7 & 0.07 \\
& & & \pm 727 & 1544 & 409 & 348 & 342 & 181 & 2.8 & 6.8 & 4.6 & 0.02 \\
\hline 190 & 15 & \multirow{2}{*}{1924} & $S_{3}$ & 1167 & 358 & 274 & 127 & -78 & -5 & -11.2 & -17.0 & -7.8 & 0.85 \\
& & & \pm 127 & 231 & 59 & 58 & 55 & 34 & 1.8 & 1 & 0.9 & 0.02 \\
& & \multirow{2}{*}{$A_{4}$} & 4095 & 2087 & 1549 & 384 & 295 & -25 & -15.0 & -52.3 & -8.3 & 0.15 \\
& & & \pm 634 & 1309 & 310 & 279 & 279 & 152 & 9.0 & 4.8 & 4.1 .5 & 0.02 \\
\hline
\end{tabular}

$A_{1}$ subcomponent with $\left(\sigma_{1}, \sigma_{2}, \sigma_{3}\right)=(15 \pm 7,6 \pm 16,6 \pm 12)$.

$A_{2}$ subcomponent with $\left(\sigma_{1}, \sigma_{2}, \sigma_{3}\right)=(16 \pm 5,9 \pm 9,11 \pm 5)$.

$S_{3}$ Gaussian component with $\left(\sigma_{1}: \sigma_{2}: \sigma_{3}\right)=(34 \pm 2,19 \pm$ $6,17 \pm 2), \varepsilon=9 \pm 5^{\circ}$.

$A_{4}$ Gaussian component with $\left(\sigma_{1}, \sigma_{2}, \sigma_{3}\right)=(64 \pm 5,46 \pm$ $14,39 \pm 4), \varepsilon=10 \pm 10^{\circ}$.

Like in HIPPARCOS sample, the Gaussian components may be identified with thin disk, $S_{2} \approx S_{3}$, and thick disk, $A_{4}$. The thin disk is subdivided in early-type stars, $A_{1}$, young disk stars, $A_{2}$, and a non discrete population of old disk stars $S_{3}-\left(A_{1} \cup A_{2}\right)$.

\section{Discussion}

MEMPHIS has demonstrated to be useful in order to identify stellar populations within nearby stars by using only statistical kinematic properties. A sampling parameter produced a hierarchical set of nested samples in order to detect significant discontinuities of their velocity distribution. The modulus of the velocity, referred to the cumulative subcentroid of the nested subsamples, provided us with a good approximation to an ideal sampling parameter. Although the method had been designed to identify normal distributions, it also provided, as a less prior information pattern, a good approach to segregate non-Gaussian populations. Entropy variations of the mixture probability allowed us to estimate the number of components or subcomponents without any prior assumption about such a number.

\subsection{Galactic components}

The detected local populations show kinematic parameters and mixing proportions that worthy improve results of Paper I. As expected, thin disk with $\left(\sigma_{1}, \sigma_{2}, \sigma_{3}\right)=(28 \pm 1,16 \pm 2,13 \pm 1)$, and thick disk with $\left(\sigma_{1}, \sigma_{2}, \sigma_{3}\right)=(65 \pm 2,39 \pm 9,41 \pm 2)$, are the basic components of our local sample. Within the thin disk, three different statistical populations were identified, with specific kinematic properties. Both of them are discrete nonGaussian populations: early-type stars, $\left(\sigma_{1}, \sigma_{2}, \sigma_{3}\right)=(12 \pm$ $31,11 \pm 1,7 \pm 1)$, and young disk stars, $\left(\sigma_{1}, \sigma_{2}, \sigma_{3}\right)=(16 \pm$ $22,14 \pm 1,14 \pm 1)$. The other subcomponent is a continuous population of old disk stars, that expands as a wing of the young ones. Its radial velocity dispersion varies in the interval $16 \leq$ $\sigma_{1} \leq 50$, but the vertical dispersion remains nearly constant at $\sigma_{3}=13$.

In the Fig. 7, the velocity ellipsoids (projected on the $V_{1} V_{2}$ plane) of the obtained populations are displayed. Both nonGaussian stellar populations, early-type stars $A_{1}$, and youngdisk stars $A_{2}$, as well as the non-continuous population of old disk stars compose the thin disk component $S_{2}$ (similar to $S_{3}$ ). The beginning of thick disk population - although mixed with old disk stars - is represented by the ellipsoid $A_{3}$. The thick disk component, $A_{4}$, is progressively build as enlarging the subsamples (points labeled as $T$ ). Let us note that the centroids of populations $A_{2}, A_{3}$ and $A_{4}$, as well as the partial thick disk populations $T$ obtained from sampling parameters $|\boldsymbol{V}| \geq 145$ are clearly aligned. This is suggesting that all the local populations, except the early-type stars $A_{1}$, are moving in the same direction with respect to the thick disk component. Therefore they are dominated by the same galactic differential movement, while $A_{1}$ presents a clearly expanding behaviour. This subcomponent can be identified with the local Gould's Belt within a distance of 300 pc (e.g. Moreno et al. 1999; Asiain et al. 1999a, Torra et al. 2000).

The Fig. 8 shows, in a bilogarithmic plot, the moments $\mu_{11}=\sigma_{1}^{2}, \mu_{33}=\sigma_{3}^{2}$, and the square of the total dispersion $\sigma^{2}$, that is, the quadratic sum of the dispersions of the three velocity components. The vertical dashed lines correspond to sampling parameter values of optimal segregations. From the pair of points $(a, b)$ up to the beginning of lines (c, d) MEMPHIS only detects a single component, that will become the thin disk (c) as enlarging the subsamples. Notice the continuous and linear trend of partials thin disk (c) and thick disk (d). When the thick disk has been completely build up (e), then the thick disk changes its slope (f).

Therefore, the extreme old disk stars are mixed with the thick disk in the range $38 \leq \sigma_{1} \leq 50$, suggesting the existence of a false intermediate population. As enlarging the subsamples, such intermediate population becomes progressively redistributed between thin and thick disk components.

Most stars with $|\boldsymbol{V}|<51 \mathrm{~km} \mathrm{~s}^{-1}$ belong to the young disk, most stars with $|\boldsymbol{V}|<131 \mathrm{~km} \mathrm{~s}^{-1}$ belong to the thin disk, and the 


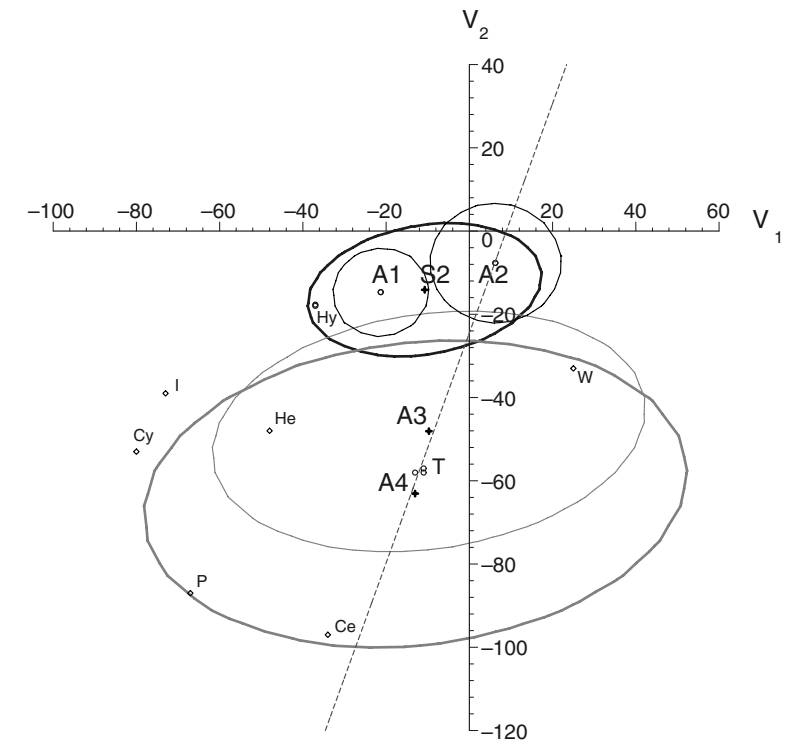

Fig. 7. Projection of velocity ellipsoids on the plane of rotationradial heliocentric velocities (rotation velocity $V_{2}$ is positive in the direction of galactic rotation, and radial velocity $V_{1}$ is positive toward the Galactic centre) for discrete populations obtained from local HIPPARCOS subsamples. Old disk stars were not obtained as a discrete stellar component, but as a broad wing of the young-disk population $A_{2}$. All together with early-type stars, $A_{1}$, they make up the thin disk component $S_{2}$ (similar to $S_{3}$ ). The subcomponent $A_{3}$ contains some old thin disk stars and a larger number of thick disk stars, that becomes the thick disk when wider local samples are considered. Notice that the populations $A_{2}, A_{3}$ and $A_{4}$, as well as the increasing thick disk populations (T), obtained from sampling parameters $|\boldsymbol{V}| \geq 145 \mathrm{~km} \mathrm{~s}^{-1}$, can be clearly fitted according a straight line. This fact is suggesting that all the local populations, except the early-type stars $A_{1}$, are moving in the same direction with respect to the thick disk component. They are dominated by a common differential galactic movement, mainly, but not only, in rotation. Some moving groups (Eggen 1971) are also displayed so that they may be associated with any of the obtained major populations. The displayed moving groups are Hyades (Hy), Wolf 630 (W), $\varepsilon$ Indi (I), $\zeta$ Herculis (He), 61 Cygni $(\mathrm{Cy}), \sigma$ Puppis (P), $\eta$ Cephei (Ce). Other moving groups composed of young disk stars, like Pleiades and Sirius (not displayed) belong to the subcomponents $A_{1}$ and $A_{2}$, within the thin disk component $S_{2}$. In particular, the subcomponent $A_{1}$ may clearly identified with the expanding local Gould's Belt structure.

remaining ones to the thick disk (those with $|\boldsymbol{V}|>209 \mathrm{~km} \mathrm{~s}^{-1}$ are few stars with extreme kinematic behaviour, probably from the halo, or with large velocity errors). However, we have shown that the sampling parameter itself is not able to deconvolve the mixture of populations. For each sampling parameter two components or subcomponents were found, and a second filtering step is needed through a bimodal mixture model.

Around $10 \%$ of moment $\mu_{12}$ is due to the velocity difference between subcentroid components by showing that the radial velocity difference is not null. Stars with smaller peculiar velocities have greater vertex deviation, as known since long time ago (e.g. Delhaye 1965). Vertex deviation is greater for stars with smaller velocity perpendicular to galactic plane. These results are also compatible with the idea that younger stars have greater vertex deviation and greater rotational velocity. The differential movement between the complete thin and thick disks produces a lag in rotation of $51 \pm 3 \mathrm{~km} \mathrm{~s}^{-1}$, as well as an small but significant lag in the radial direction of $4 \pm 3 \mathrm{~km} \mathrm{~s}^{-1}$, as it was found in Paper I. Although early-type stars expand with reference to young disk stars with radial differential velocity of about $28 \pm 9 \mathrm{~km} \mathrm{~s}^{-1}$.

By comparing HIPPARCOS and CNS3 catalogues, we have found entropy maxima, stellar populations and kinematic parameters very similar, though errors are much smaller for HIPPARCOS. This situation is produced even though the samples are different in their conception and limiting distances, $300 \mathrm{pc}$ for HIPPARCOS and $25 \mathrm{pc}$ for CNS3. We conclude that both samples give the same qualitative portrait of the solar neighbourhood.

\subsection{Symmetries}

We are able to interpret the results according to the dynamic models described in Sect. 2, according to particular symmetry assumptions. For HIPPARCOS sample, thin and thick disk components have non-null vertex deviation. This result agrees with authors who find not negligible vertex deviation of all stellar populations (Dehnen 1998). Then the stellar system model would not be consistent with the axial symmetry hypothesis and, at least, point-axial symmetry would have to be assumed, allowing barred or spiral features (Mühlbauer \& Dehnen 2003). Nevertheless, for CNS3 samples the vertex deviation of thick disk component vanishes. In fact negligibility of vertex deviation is possible for old stars or thick disk at lower scale heights, and it depends on star age. It becomes null after a long time (e.g. Gomez et al. 1997).

As discussed in Paper I, the non-null values of $\mu_{111}$ and $\mu_{122}$ for the global samples (Tables 8, 9, 11 and 12) point out the existence of a non-vanishing radial differential movement of components (Tables 3 and 5). On the other hand, nearly null values of odd-order moments in the $z$-velocity component are suggesting the existence of a Galactic plane of symmetry, and that the star samples are very close to it.

As we had commented in Sect. 2, the axiallity index $I=$ $\mu_{12} / \mu_{22}$ can be used as a measure of the non-cylindrical behaviour. Now we shall use not the galactocentric coordinates system $(\Pi, \Theta, Z)$, with $\Pi$ pointing to the Galactic anticentre, but the heliocentric $\left(V_{1}, V_{2}, V_{3}\right)$ system, with $V_{1}$ toward the Galactic centre. The axial dependency was related to the mean radial velocity according to Eq. (2), now written as follows,

$v_{1}-v_{1}^{(\mathrm{cyl})}=\frac{\mu_{12}}{\mu_{22}} \Theta_{0}$.

On the other hand, thick disk stars (Fig. 7) show a decreasing trend of vertex deviation as increasing the sampling parameter $\left(|\boldsymbol{V}|_{\max }=125,145,165,190,209 \mathrm{~km} \mathrm{~s}^{-1}\right)$, which is related with a trend to axial-symmetry. Then, if we plot the galactocentric rotation mean velocity $\Theta_{0}$ against the differential radial mean velocity referred to a cylindrical system, $v_{1}-v_{1}^{\text {(cyl) }}$, the rotation mean velocity of an hypothetical axially symmetric thick disk can be extrapolated, as shown in Fig. 8 (left), leading to a value $\Theta_{0}=144 \pm 2 \mathrm{~km} \mathrm{~s}^{-1}$. Moreover, if we assume that such an extreme thick disk is nearly in steady state, which is a plausible 

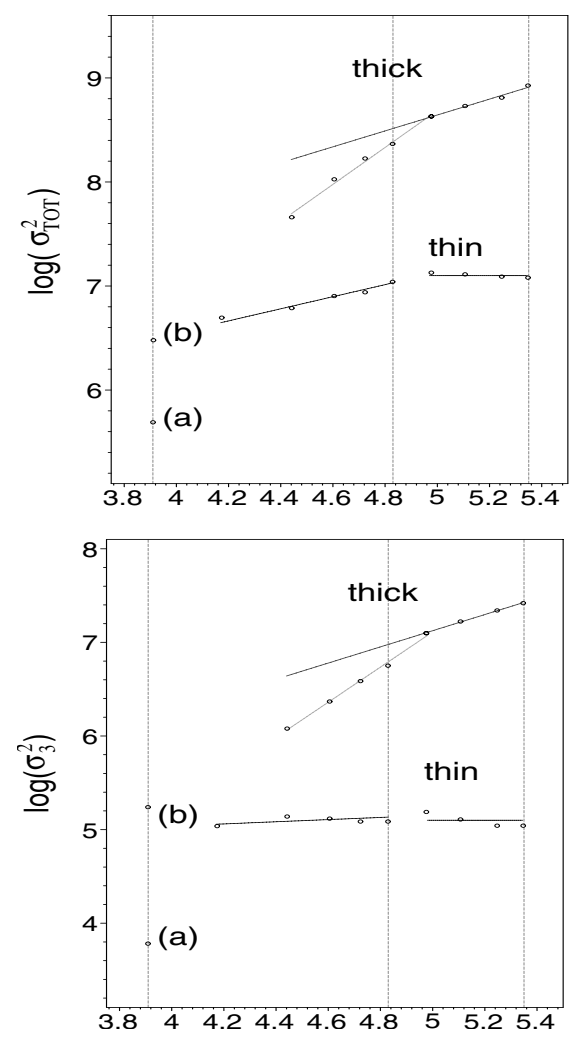

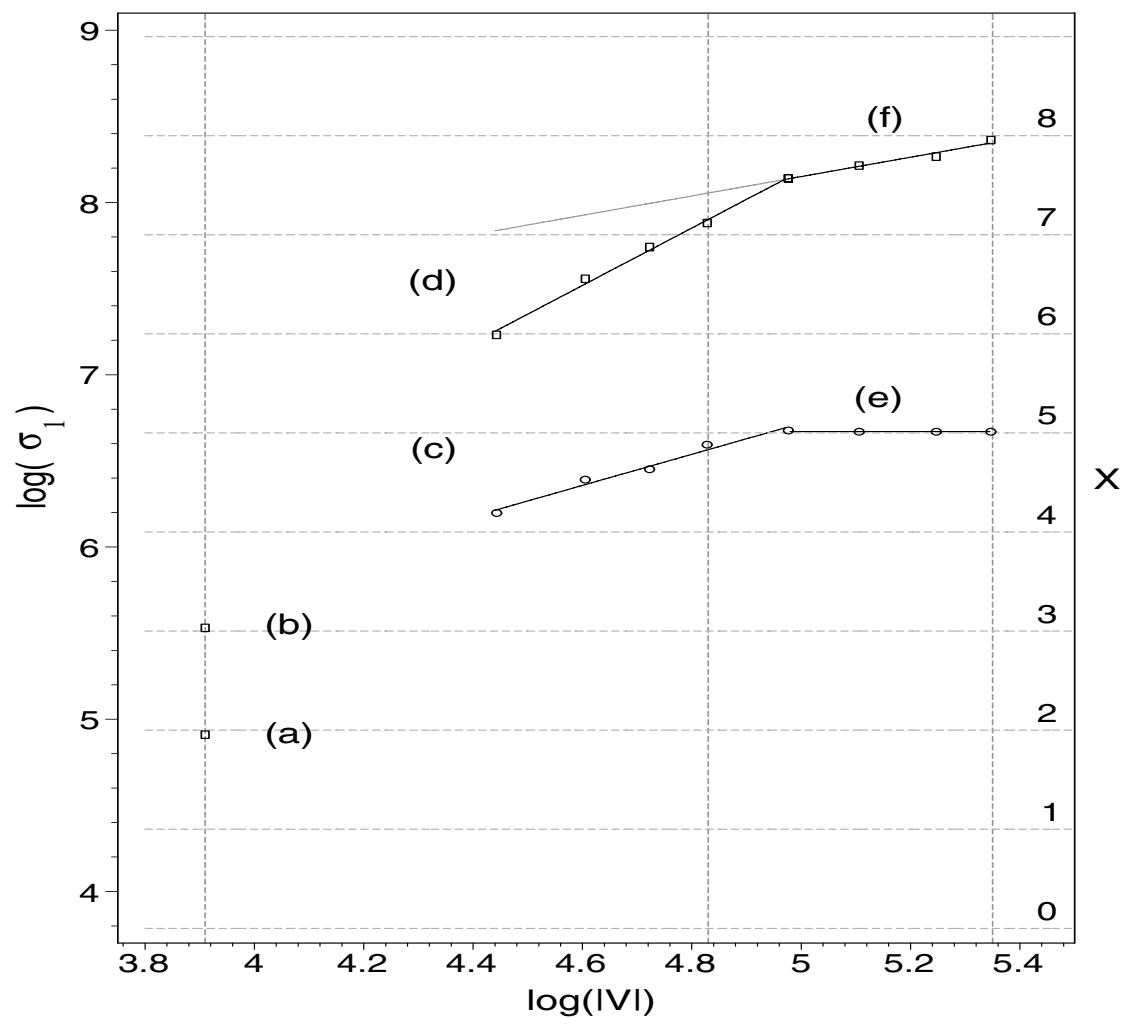

Fig. 8. The figure on the right shows, in a bilogarithmic plot, the trend between the moment $\sigma_{1}^{2}$ and the sampling parameter $P=|\boldsymbol{V}|_{\max }$, for populations segregated from several nested HIPPARCOS subsamples (dots). The dashed vertical lines indicate optimal segregations according to MEMPHIS algorithm. The first significant segregation was obtained by $\ln P=3.9\left(P=51 \mathrm{~km} \mathrm{~s}^{-1}\right)$, leading to a) early-type and b) young disk populations. From $\ln P$ between 3.9 to $4.4\left(P\right.$ from 51 to $\left.85 \mathrm{~km} \mathrm{~s}^{-1}\right)$ the method only detected a single population: the seed of the thin disk c). From $\ln P=4.4$ to 4.8 ( $P$ between 85 and $125 \mathrm{~km} \mathrm{~s}^{-1}$ ) an intermediate population d) was segregated, as an independent population from c). It was the beginning of the thick disk, overlapped with old disk stars. When the complete thin disk e) was build, for ln $P$ between 5 and 5.4 ( $P$ from 145 to $210 \mathrm{~km} \mathrm{~s}^{-1}$ ), the thick disk f) became clearly identified. The continuous dark lines represent the continuous evolution of thin and thick disk populations. The slopes of the regression lines are c) 0.90 and f) 0.86 , which is extrapolated (grey line) in order to isolate thick disks stars from d). A similar trend was fulfilled by other velocity components. Left hand side figures show the total dispersion $\sigma^{2}$ and $\sigma_{3}^{2}$ against the sampling parameter. For the total velocity dispersion, the slopes of regression lines are 0.63 (increasing thin) and 0.76 (thick). On the other hand, horizontal dashed lines are values of $x$ in the equation $\sigma_{1}=6.6\left(\frac{4}{3}\right)^{x}$, for natural values of $x$. The level $x=0$ represents a hypothetical spherical population with $\sigma_{1}=\sigma_{2}=\sigma_{3}=6.6$, similar to the $z$-velocity dispersion of the earlier stars. Early-type a) and young disk stars b) have levels $x=2$ and 3. The increasing thin disk component c) emerges from $x=3$ to 5 , while the continuous old disk stars are in the range from $x=3$ to 7 . The foregoing populations make up the thin disk component e), at level $x=5$. Finally, from $x=7$ onward, the local thick disk component alone f) is detected, up to level $x=8$.

assumption for the oldest stars, then we can admit a null radial mean velocity for the oldest thick disk (Chiba \& Beers 2000; Soubiran et al. 2003).

Similarly, Fig. 8 (right) displays the location of young disk population $A_{2}$, as well as samples of the progressive old disk stars, up to the complete local thick disk $A_{4}$, which are clearly aligned in the plane of radial-rotation heliocentric velocities $V_{1} V_{2}$. Their regression line is extrapolated up to reach the heliocentric rotation velocity of the foregoing extreme thick disk, $V_{2}=-76 \pm 2 \mathrm{~km} \mathrm{~s}^{-1}$, with zero radial velocity (by assuming a solar mean rotation velocity $\Theta_{0}=220 \mathrm{~km} \mathrm{~s}^{-1}$ ). Hence the heliocentric radial velocity of such axisymmetric population with no radial movement may be estimated about $V_{1}=-18 \pm 1 \mathrm{~km} \mathrm{~s}^{-1}$ referred to the Sun, which is quite similar to the radial velocity of early-type stars $A_{1}$ and thick disk stars $A_{4}$. Therefore, the point-axial symmetry assumption allow us to predict an approximated radial galactocentric velocity of the LSR of $\Pi_{0}=8 \pm 2 \mathrm{~km} \mathrm{~s}^{-1}$ toward the Galactic centre. Therefore, the conjectured expansion of Gould's Belt now becomes a contraction of most disk stars, since the former have a very small radial galactocentric velocity.

\subsection{Small and large-scale structures}

Recently Famaey et al. (2005) have published an analysis of a larger complementary sample of HIPPARCOS stars with radial velocities. Their results in the region where their method is consistent with this paper give results in very good agreement with the present results. Moreover our results clarify the question about the radial solar motion referred to a point with no net radial motion. In their total sample of 6030 stars, a large scale structure of the velocity distribution was found, which was composed of three stellar groups. By one hand the high-velocity stars (HV, 401 stars), similar to our thick disk 

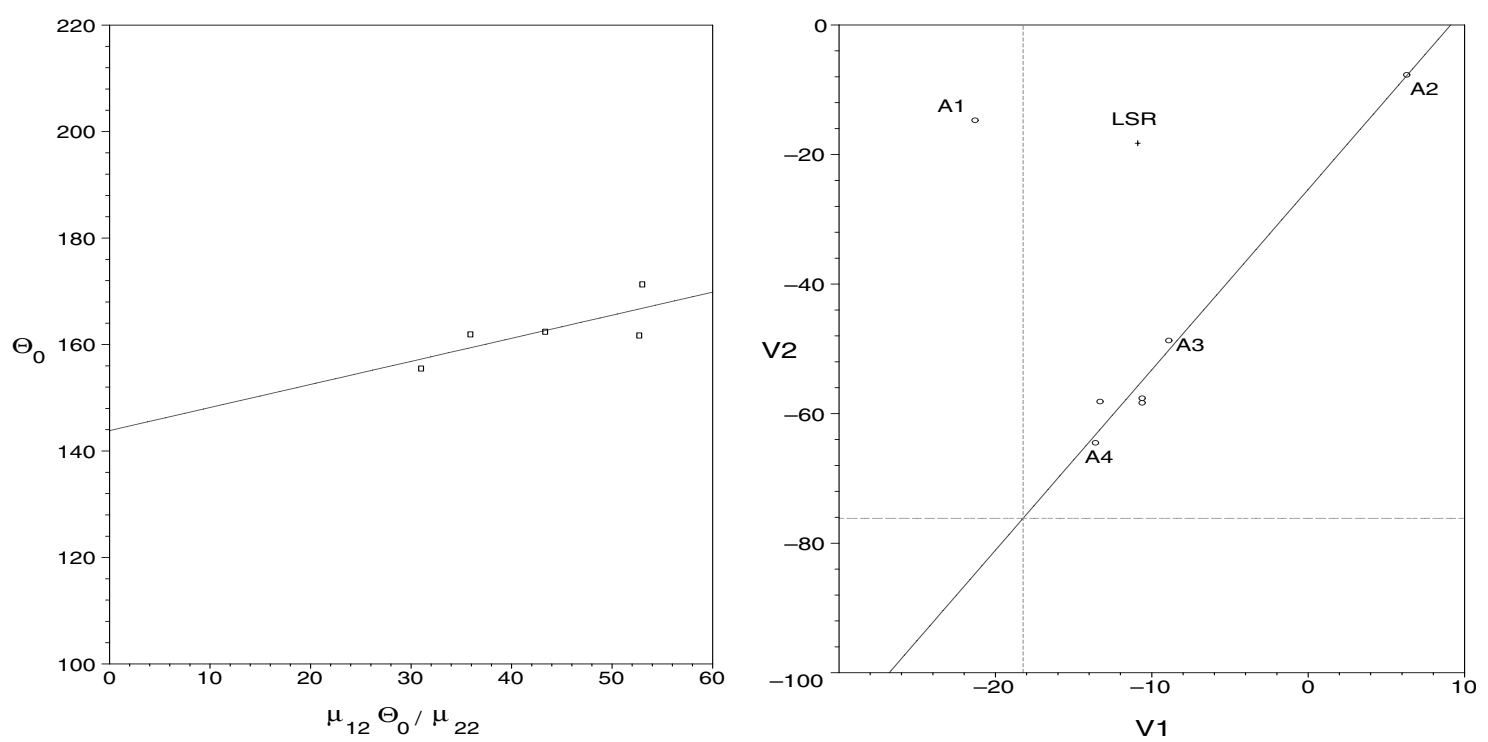

Fig. 9. The figure on the left shows how the mean rotation velocity $\Theta_{0}$ and the excess of mean radial velocity, referred to an axial system, are related, according to a point-axial system. This excess explicitly depends on the vertex deviation of the velocity ellipsoid in the way $v_{1}-v_{1}^{\text {(cyl) }}=\mu_{12} \Theta_{0} / \mu_{22}$. We can assume that the oldest thick disk population follows the trend toward zero vertex deviation, with a vanishing mean radial movement. In other words, the extreme thick disk would be nearly an axisymmetric stellar system. The plotted points correspond to several increasing thick disk samples, whose kinematic parameters show a continuous dependence on the sampling parameter $\left(|\boldsymbol{V}|_{\max }=\right.$ $125,145,165,190,209 \mathrm{~km} \mathrm{~s}^{-1}$ ). The continuous line is the regression curve of the points, that intersects the vertical axis at $\Theta_{0}=144 \pm 2 \mathrm{~km} \mathrm{~s}{ }^{-1}$, with a slope of $0.44 \pm 0.05$. Thus, the extrapolated value may be assumed as the approximated rotation velocity of the oldest thick disk population. The figure on the right displays the location of young disk population $A_{2}$, and older populations, from $A_{3}$ to the local thick disk $A_{4}$, which share a common direction in the plane of radial-rotation heliocentric velocities $\left(V_{1} V_{2}\right)$. Their straight line fit $\left(V_{2}=-25.4_{(0.6)}+2.79_{(0.06)} V_{1}\right)$ is extrapolated up to reach the heliocentric rotation velocity $-76 \pm 2 \mathrm{~km} \mathrm{~s}^{-1}$ for the extreme thick disk with zero radial velocity (by assuming a local mean rotation velocity $\Theta_{0}=220 \mathrm{~km} \mathrm{~s}^{-1}$ ). Hence the heliocentric radial velocity of such axisymmetric population, with no radial galactic movement, may be estimated about $-18 \pm 1 \mathrm{~km} \mathrm{~s}^{-1}$, which is very close to the radial velocity of earlier stars $A_{1}$.

component $A_{4}$, and, on the other hand, the group of young kinematics stars (Y, 413 stars) and the group of "normal" stars (5216 stars), which compose our thin disk component $S_{3}$. A further analysis of the small-scale structure of their "normal" stars revealed the existence of three subgroups over a smooth background: Hercules stream (He, 529 stars) with kinematical properties of the hottest thin disk, like an important part of the subcomponent $A_{3}$; Hyades-Pleiades supercluster (HyPl, 392 stars), with kinematics corresponding to the subcomponent $A_{1}$; and the Sirius moving group ( $\mathrm{Si}, 268$ stars), that together with the smooth background (4027 stars), may be identified with our $A_{2}$ subcomponent.

In addition, Soubiran \& Girard (2005) have analysed the chemical distribution of solar neighbourhood stars, and they compare their kinematics with the stellar groups of Famaey et al. (2005). Their results are also completely consistent with our 3D-velocity based analysis. Thin disk, thick disk and Hercules stream were described according to three ellipsoidal distributions. They have found thin and thick disks well separated, although greatelly overlapped in metallicity. On the other hand, Hercules stream was found to be mainly made of thin disk stars, with a few thick disk stars. The authors suspect that previous studies had included this group within the thick disk. This situation clarifies the description of our subcomponent $A_{3}$ - containing the Hercules group - which was still composed of $32 \%$ of thin disk stars, and that could be prematurely interpreted as the thick disk. Moreover, in order to study the thin disk small-scale structure, they subtracted the Hercules stellar group from the thin disk. A different chemical and kinematical behaviour was obtained between the super metal rich part and the rest of the thin disk. The half of the thin disk stars, with $[\mathrm{Fe} / \mathrm{H}]>+0.20$, had motion consistent with that of the Hyades-Pleiades supercluster, the one that Famaey et al. (2005) propose to be originated from a common large molecular cloud, radially perturbed by a spiral wave. Such result is clearly consistent with the segregation of thin disk's subcomponent $A_{1}-$ that we had associated with the Gould's Belt structure - and the remaining subcomponent $A_{2}$.

Let us note that our method is devoted to the study of the large-scale structure of the velocity distribution, in order to assess the main dynamical trend of solar neighbourhood stars. We avoid to manually clean any stellar component from an specific group of stars, since the Central Limit Theorem is then no more fulfilled. On the other hand, we needed the maximum number of stars to carry out a full statistical analysis of star's kinematical properties. Such analysis allowed us to determine a common trend of half of the thin disk (subcomponent $A_{2}$ ) and the increasing thick disk, which share a common movement direction referred to a no net radial motion point, which is the claimed reference point by Famaey et al. (2005). Along that direction, as the average age of stars gets older and their velocity dispersions increase, the mean radial and rotation velocities, as well as the vertex deviation, decrease up to reach a point in the velocity space which moves in a pure circular 
Table 6. Comparative of values with TBLL $\sigma_{1}(x)=6.6\left(\frac{4}{3}\right)^{x}$.

\begin{tabular}{ccccc}
\hline \hline$n$ & $\sigma_{1}$ from TBLL & $\sigma_{1}$ from MEMPHIS & MEMPHIS component & Stellar populations \\
\hline 0 & 6.6 & - & - & spherical population at birth \\
2 & 12 & 12 & $A_{1}$ & early-type stars \\
3 & 16 & 16 & $A_{2}$ & young disk stars \\
5 & 28 & 28 & $S_{3}$ & thin disk \\
7 & 50 & 50 & $A_{3}$ & thick disk + old disk stars \\
8 & 66 & 65 & $A_{4}$ & thick disk \\
\hline
\end{tabular}

orbit, the one consistent with the axisymmetric hypothesis. Thus, the cumulative effect over galactic rotations of a dynamic perturbation, such a galactic bar or a spiral wave, would produce the rupture of the axial symmetry at star formation time, and would provide the younger stellar populations with a net radial motion up to reach the dynamic equilibrium, associated with an elliptical orbit of the centroid around the galactic center. As disk populations get hotter, the trend towards axisymmetry arises again. The region of thin disk's velocities where the axiallity would be disrupted corresponds to the $A_{1}$ subcomponent (around Hyades-Pleiades supercluster), in whose neighbourhood above authors have found significant dynamical streams of stars, with quite mixed ages, and other authors (Skuljan et al. 1999) have described a three branch structure, which is distributed along the major axis of our thin disk component $S_{3}$. Therefore our results, under the framework of a superposition of point-axial Candrasekhar's systems, stress a dynamical origin for such transient features.

\subsection{A Titius-Bode-like law}

A more detailed analysis is shown in Fig. 8, where the radial velocity dispersion is represented against the sampling parameter $P=|\boldsymbol{V}|_{\max }$, in a bilogarithmic plot. If we compare the radial velocity dispersions $\sigma_{1}$ of HIPPARCOS populations (Table 3), we get, quite accurately, the following ratios,

$$
\frac{\sigma_{1}\left(A_{2}\right)}{\sigma_{1}\left(A_{1}\right)} \sim \frac{4}{3}, \frac{\sigma_{1}\left(S_{3}\right)}{\sigma_{1}\left(A_{2}\right)} \sim\left(\frac{4}{3}\right)^{2}, \frac{\sigma_{1}\left(A_{3}\right)}{\sigma_{1}\left(S_{3}\right)} \sim\left(\frac{4}{3}\right)^{2}, \frac{\sigma_{1}\left(A_{4}\right)}{\sigma_{1}\left(A_{3}\right)} \sim \frac{4}{3} .
$$

Therefore, it would seem reasonable to describe the radial dispersion of obtained populations through a law $\sigma_{1} \propto\left(\frac{4}{3}\right)^{x}$. For example,

$\sigma_{1}(x)=6.6\left(\frac{4}{3}\right)^{x}$

We have taken the radial dispersion of the thin disk $S_{3}, \sigma_{1}=$ 28 , as a reference value, so that the level $x=0$ is associated with the closest value to the lowest vertical velocity dispersion $\sigma_{3}\left(A_{1}\right)$ obtained from our subsamples, in order to represent a hypothetical population at birth, with spherical distribution. In any case, it is an arbitrary value. Hence, the populations contained in our HIPPARCOS velocity sample can be described from natural values of $x$ according to the Table 6 .

It is well known, since eighteenth century, that the empiric Titius-Bode distribution determines the radii of orbits in a
Keplerian planetary system depending on a natural number, although the possibility of an underlying physical explanation for the observed distribution remains open (Lynch 2003). Now we have obtained a similar relation between radial velocity dispersions of stellar populations and natural numbers. For similarity, we shall name Eq. (17) Titius-Bode-like law (hereafter TBLL). Such results are consistent with Galactic formation models that predict some quasi-continuous stellar populations (see e.g. review from Majewski 1993) in the sense that the continuity is constricted by $\sigma_{1}$ levels of TBLL.

The physical meaning of the variable $x$ involved in the TBLL may be related with the average epicycle energy $E_{R} \sim$ $\sigma_{1}^{2}$ of the stars (Lacey 1984; Jenkins 1992), representative of the disk heating process. It shows continuity from $x=3$ to 5 for the thin disk, and from 7 to 8 for the thick disk, but discreteness from $x=2$ to 3 between early-type and young stars, and from 5 to 8 between thin and thick disk components. For the thin disk, for example, the level $x=5$ should represent the saturation point of maximum velocity dispersion, likely corresponding to the limited predicted by the observed wavenumber of spiral structure of the Milky Way, while the discontinuity at $|\boldsymbol{V}|_{\max } \sim 145 \mathrm{~km} \mathrm{~s}^{-1}$, from $x=5$ to 7.5 , indicates an abrupt jump in the average energy, that was produced when the thick disk was formed about $10 \pm 1 \mathrm{Gyr}$ ago (e.g. Quillen \& Garnett 2001). On the other hand, for values $x \leq 3$ our model could not detect continuous variations of the velocity distribution, since the velocity distribution is not enough homogeneous, and it mainly reflects star streams or moving groups.

Usually the disk heating process is described through the age-velocity law for stellar scattering (e.g. Wielen 1977), given by the approximate equation

$\sigma(t)^{n}=\sigma\left(t_{0}\right)^{n}+C_{v} t$

where $C_{v}$ is the apparent diffusion coefficient. The foregoing diffusion equation may also be written in the form,

$\sigma(\tau)=\sigma\left(t_{0}\right) \tau^{\alpha}, \quad$ with $\quad \alpha=\frac{1}{n}, \tau=1+\frac{t}{t_{0}}, t_{0}=\frac{\sigma\left(t_{0}\right)^{n}}{C_{v}}$.

It may be either applied to radial or vertical velocity dispersions (Jenkins 1992; Kroupa 2002), as well as to the total velocity dispersion of the peculiar velocities.

In the Fig. 8 we have shown a similar dependence of $\ln \sigma$ versus the sampling parameter $|\boldsymbol{V}|_{\max }$ defining the nested subsamples. The greater the sampling parameter, the greater the average age of merged stars. Thus, a the relationship between $|\boldsymbol{V}|_{\max }$ and age can be approximately established. 
The variable $\tau$ of Eq. (19) measures the age of the stars relatively to an arbitrary quantity $t_{0}$ (it may be interpreted as the necessary time for a population to reach a dispersion $2^{\alpha} \sigma\left(t_{0}\right)$ from the initial value $\sigma\left(t_{0}\right)$ ). In the solar neighbourhood, depending on the heating mechanism (scattering only by clouds or together with a spiral wave, with or without accretion, etc.), some accepted values for $\sigma\left(t_{0}\right)$ and $\alpha$ are $15 \mathrm{~km} \mathrm{~s}^{-1}, 0.2$ (Lacey 1991), $8 \mathrm{~km} \mathrm{~s}^{-1}, 0.33$ (Binney et al. 2000), $10 \mathrm{~km} \mathrm{~s}^{-1}, 0.5$ (Wielen et al. 1996), and $t_{0}$ may vary from 0.01 to $0.45 \mathrm{Gyr}$ (Asiain et al. 1999b). Hence, for stars with ages of a few Gyr, like most of the solar neighbourhood, the variable $\tau$ can be used simply as the relative age to $t_{0}$. The continuous line (c) in Fig. 8 , that fits the progressively heated thin disk, has a slope $\sim 0.90$, hence, according to Eq. (19) (for radial velocities, $\sigma=\sigma_{1}$ and $\left.\alpha=\alpha_{1}\right)$ we can write $\sigma_{1}^{2} \propto \tau^{2 \alpha_{1}} \propto|\boldsymbol{V}|_{\max }^{0.90}$, hence we can estimate $|\boldsymbol{V}|_{\max } \propto \tau^{\frac{\alpha_{1}}{0.45}}$. Notice that the regression line (f) for the thick disk has a slope very similar, 0.86 , which would indicate an intriguing similar heating rate for the thick disk stars. Similarly, the slopes of the regression lines for the total velocity dispersion $\sigma^{2}$ (Fig. 8, upper-left side) are $\sim 0.64$ (thin disk) and $\sim 0.76$ (thick disk), once more very similar. If we had accepted the most recent value $\alpha=0.33$ for the law velocity-age (Binney et al. 2000), then we should have nearly the age estimation through our sampling parameter,

$|\boldsymbol{V}|_{\max } \propto \tau^{\frac{\alpha}{0.32}} \sim \tau$.

On the other hand, in Eq. (17) we had adopted an initial value for the radial velocity dispersion $\sigma_{1}=6.6$, for a spherical distribution of stars at birth, with an initial total dispersion $\sigma=11.5 \approx 6.6 \sqrt{3}$, which is consistent with above referred values. Thus, if we compare intervals with continuous variation of Eqs. (17) and (19) for the radial velocity dispersion $\sigma_{1}$, and we assume the approximate relationship of Eq. (20), it is possible to roughly estimate $\alpha_{1} \sim 0.45$ and $x \sim 1.5 \ln \tau$.

Then, by reading Fig. 8 from right to left, and according to Eq. (19), a continuous heating of the thin disk (c) from $x=5$ (its origin, after the formation of the thick disk) to 0 (actual date) during $10 \mathrm{Gyr}$ (its approximate age) would provide an estimated value $t_{0}=0.4 \mathrm{Gyr}$. Also, the time before the discontinuity between thin and thick disk could be estimated from $x=8$ to 7.5 along (f), leading to an age of $13.8 \mathrm{Gyr}$ for the thick disk. The foregoing roughly estimations are totally consistent with the actual portrait of the galactic disk (e.g. Binney et al. 2000; Fuchs et al. 2001; Soubiran \& Girard 2005) and are only an example of the information that can be obtained only from kinematic data.

Acknowledgements. The authors wish to thank Professor J. J. de Orús and Dra. M. A. Català-Poch who introduced them into this exciting field.

\section{References}

Alcobé, S. 2001, Ph.D. Thesis, Universitat de Barcelona, Barcelona Alcobé, S., \& Cubarsi, R. 2001, ASP-CS, 230, 43

Alcobé, S., \& Cubarsi, R. 2004, ASP-CS, 316, 234

Asiain, R., Figueras, F., Torra, J., \& Chen, B. 1999a, A\&A, 341, 427
Asiain, R., Figueras, F., \& Torra, J. 1999b, A\&A, 350, 434

Beers, T., \& Sommer-Larsen J. 1995, ApJS, 96, 175

Binney, J., Dehnen, W., Houk, N., Murray, C. A., \& Penston, M. J. 1997, in Hipparcos-Venice'97, ESA SP-402, 473

Binney, J., Dehnen, W., \& Bertelli, G. 2000, MNRAS, 318, 658

Català-Poch, M. A. 1972, Urania, N. 275, Tarragona

Chandrasekhar, S. 1942, in Principles of Stellar Dynamics (New York: Dover Publications Inc.)

Chiba, M., \& Beers, T. C. 2000, AJ, 119, 2843

Cubarsi, R. 1990, AJ, 99, 1558

Cubarsi, R. 1992, AJ, 103, 1608

Cubarsi, R., \& Alcobé, S. 2004, A\&A, 427, 131

Dehnen, W. 1998, AJ, 115, 2384

Dehnen, W., \& Binney, J. J. 1998, MNRAS, 298, 387

Delhaye, J. 1965, in Galactic Structure, ed. A. Blaaw, \& M. Schmidt (Chicago: University of Chicago Press)

Eggen, O.J. 1971, PSAP, 83, 251

Erickson, R. R. 1975, ApJ, 195, 343

ESA 1992, The Hipparcos Input Catalogue, ESA SP-1136

ESA 1997, The Hipparcos Catalogue, ESA SP-1200

Famaey, B., Jorissen, A., Luri, X., et al. 2005, A\&A, 430, 165

Fuchs, B., Dettbarn, C., Jahreiß, H., \& Wielen, R. 2001, ASP-CS, 228, 235

Gliese, W., \& Jahreiß, H. 1991, Third Catalogue of Nearby Stars, Astronomisches Rechen-Institut, Heidelberg

Gómez, A.E., Grenier, S., Udry, S., et al. 1997, in Hipparcos-Venice'97, ESA SP-402, 621

Jenkins, A. 1992, MNRAS, 257, 620

Juan-Zornoza, J. M. 1995, Ph.D. Thesis. Universitat de Barcelona, Barcelona

King, I. R. 1995, in Stellar Populations, IAU Symp., 164, 337

Kroupa, P. 2002, MNRAS, 330, 707

Lacey, C. G. 1984, MNRAS, 208, 687

Lacey, C. G. 1991, in Dynamics of disk galaxies, Varberg Castle, Sweden, p. 257

Lynch, P. 2003, MNRAS, 341, 1174

Majewski, S. R. 1993, ARA\&A, 31, 575

Moreno, E., Alfaro, E. J., \& Franco, J. 1999, ApJ, 522, 276

Mühlbauer G., \& Dehnen W. 2003, A\&A, 401, 975

Ojha, D. K., Bienaymé, O., Robin, A. C., \& Mohan, V. 1994, A\&A, 290, 771

Ojha, D. K., Bienaymé, O., Robin, A. C., Crézé, M., \& Mohan, V. 1996, A\&A, 311, 456

Orús, J. J. de 1952, Doctoral Thesis, Collectanea Mathematica, Vol. V(2), Universitat de Barcelona, Barcelona

Papoulis, A. 1989, in Probability, Random Variables, and Stochastic Processes, MacGraw-Hill, Singapore

Press, W. H., Teukolsky, S. A., Vetterling, W. T., \& Flannery, B. P. (1992), Numerical Recipes in Fortran (Cambridge: Cambridge University Press), 279

Quillen, A. C., \& Garnett, D. R. 2001, ASP-CS, 230, 87

Sala, F. 1990, A\&A, 235, 85

Sanz-Subirana, J., \& Català-Poch, M. A. 1987, in 10th ERAM of the IAU, 4, 267

Skuljan, J., Hearnshaw, J. B., \& Cottrell, P. L. 1999, MNRAS, 308, 731

Soubiran, C., Bienaymé , O., \& Siebert, A. 2003, A\&A, 398, 141

Soubiran, C., \& Girard, P. 2005, A\&A, 438, 139

Torra, J., Fernández, D., \& Figueras, F. 2000, A\&A, 359, 82

Wielen, R. 1977, A\&A, 60, 263

Wielen, R., Fuchs, B., \& Dettbarn, C. 1996, A\&A, 314, 448

Wyse, R. F. G., \& Gilmore, G. 1995, AJ, 110, 2771 


\section{Online Material}


S. Alcobé and R. Cubarsi: Disk populations from HIPPARCOS kinematic data, Online Material p 2

Table 7. Central moments and cumulants for HIPPARCOS heliocentric sample, limited by $|\boldsymbol{V}|=51 \mathrm{~km} \mathrm{~s}^{-1}$, with 10195 stars.

\begin{tabular}{|c|c|c|c|c|c|}
\hline $\bar{i}$ & $V_{i}$ & & $i j k$ & $\mu_{i j k}$ & \\
\hline 1 & -7.43 & \pm .19 & & & \\
\hline 2 & -11.18 & .13 & 111 & 2414.01 & \pm 133.17 \\
\hline \multirow[t]{2}{*}{3} & -6.42 & .11 & 112 & 124.02 & 61.18 \\
\hline & & & 122 & 616.82 & 45.47 \\
\hline$i j$ & $\mu_{i j}$ & & 222 & 476.23 & 68.22 \\
\hline 11 & 384.48 & \pm 4.87 & 113 & 275.90 & 34.12 \\
\hline 12 & 48.59 & 2.37 & 123 & 59.64 & 29.25 \\
\hline 22 & 174.57 & 2.49 & 223 & 91.52 & 37.06 \\
\hline 13 & -3.07 & 2.15 & 133 & 354.14 & 40.22 \\
\hline 23 & -2.25 & 1.57 & 233 & 231.35 & 32.74 \\
\hline 33 & 117.18 & 2.18 & 333 & 220.78 & 57.56 \\
\hline$i j k l$ & $\mu_{i j k l}$ & & $i j k l$ & $\kappa_{i j k l}$ & \\
\hline 1111 & 389505.86 & \pm 8920.82 & 1111 & -53973.13 & \pm 11028.73 \\
\hline 1112 & 36120.20 & 2825.80 & 1112 & -19928.17 & 3454.60 \\
\hline 1113 & 1565.02 & 2591.00 & 1113 & 5100.74 & 2973.43 \\
\hline 1122 & 59566.47 & 1437.56 & 1122 & -12273.37 & 2333.03 \\
\hline 1123 & -760.31 & 894.78 & 1123 & 401.35 & 1097.42 \\
\hline 1133 & 47210.88 & 1341.73 & 1133 & 2139.26 & 1945.77 \\
\hline 1222 & 9427.84 & 1602.30 & 1222 & -16019.75 & 1850.65 \\
\hline 1223 & -102.54 & 673.21 & 1223 & 650.90 & 783.23 \\
\hline 1233 & 2991.07 & 683.06 & 1233 & -2716.63 & 783.50 \\
\hline 1333 & 556.81 & 1361.56 & 1333 & 1634.38 & 1433.46 \\
\hline 2222 & 93835.38 & 3809.14 & 2222 & 2415.54 & 4096.61 \\
\hline 2223 & 1060.50 & 1317.91 & 2223 & 2237.02 & 1404.33 \\
\hline 2233 & 25202.44 & 965.39 & 2233 & 4737.05 & 1176.77 \\
\hline 2333 & 1213.02 & 1202.16 & 2333 & 2002.76 & 1245.98 \\
\hline 3333 & 62223.93 & 2875.00 & 3333 & 21031.87 & 3008.22 \\
\hline
\end{tabular}

Table 8. Central moments and cumulants for HIPPARCOS heliocentric sample limited by $|\boldsymbol{V}|=131 \mathrm{~km} \mathrm{~s}^{-1}$, with 13,315 stars.

\begin{tabular}{|c|c|c|c|c|c|}
\hline $\bar{i}$ & \multicolumn{2}{|l|}{$\overline{\overline{V_{i}}}$} & \multicolumn{3}{|c|}{$\mu_{i j k}$} \\
\hline 1 & -10.35 & \pm .27 & & & \\
\hline 2 & -17.05 & .18 & 111 & 2497.22 & \pm 983.54 \\
\hline \multirow[t]{2}{*}{3} & -7.24 & .13 & 112 & -6279.45 & 386.69 \\
\hline & & & 122 & -1150.26 & 298.98 \\
\hline$i j$ & $\mu_{i j}$ & & 222 & -8662.00 & 448.13 \\
\hline 11 & 944.63 & \pm 14.77 & 113 & -122.28 & 328.78 \\
\hline 12 & 99.71 & 6.83 & 123 & 119.91 & 170.32 \\
\hline 22 & 424.50 & 7.62 & 223 & -299.52 & 194.91 \\
\hline 13 & -13.43 & 5.63 & 133 & 50.37 & 226.55 \\
\hline 23 & 10.83 & 4.03 & 233 & -2265.19 & 185.45 \\
\hline 33 & 240.36 & 5.28 & 333 & 30.14 & 325.81 \\
\hline$i j k l$ & $\mu_{i j k l}$ & & $i j k l$ & $\kappa_{i j k l}$ & \\
\hline 1111 & 3796357.00 & \pm 132175.24 & 1111 & 1119360.12 & \pm 140732.81 \\
\hline 1112 & 183822.64 & 36654.71 & 1112 & -98735.20 & 39142.15 \\
\hline 1113 & -74239.09 & 36496.32 & 1113 & -36174.01 & 37726.85 \\
\hline 1122 & 631699.04 & 20993.54 & 1122 & 210815.36 & 25016.57 \\
\hline 1123 & 7438.00 & 11370.37 & 1123 & -117.43 & 12078.38 \\
\hline 1133 & 422382.55 & 18225.00 & 1133 & 194965.64 & 20125.78 \\
\hline 1222 & 118875.61 & 21987.01 & 1222 & -8101.72 & 22882.96 \\
\hline 1223 & -11807.29 & 9077.28 & 1223 & -8265.72 & 9437.38 \\
\hline 1233 & 18538.18 & 7942.35 & 1233 & -5136.62 & 8234.74 \\
\hline 1333 & -30101.79 & 15112.02 & 1333 & -20416.04 & 15312.08 \\
\hline 2222 & 953476.55 & 43783.72 & 2222 & 412864.80 & 45195.12 \\
\hline 2223 & 23912.47 & 15755.64 & 2223 & 10115.51 & 16059.13 \\
\hline 2233 & 216543.68 & 11228.97 & 2233 & 114273.29 & 11945.19 \\
\hline 2333 & -3100.13 & 13918.00 & 2333 & -10912.29 & 14031.04 \\
\hline 3333 & 428653.51 & 31433.98 & 3333 & 255328.64 & 31739.67 \\
\hline
\end{tabular}


S. Alcobé and R. Cubarsi: Disk populations from HIPPARCOS kinematic data, Online Material p 3

Table 9. Central moments and cumulants for HIPPARCOS heliocentric sample limited by $|\boldsymbol{V}|=209 \mathrm{~km} \mathrm{~s}^{-1}$, with 13530 stars.

\begin{tabular}{|c|c|c|c|c|c|}
\hline $\bar{i}$ & \multicolumn{2}{|l|}{$\overline{\bar{V}}$} & \multicolumn{3}{|c|}{$\overline{\mu_{i j k}}$} \\
\hline 1 & -10.86 & \pm .28 & & & \\
\hline 2 & -18.25 & .20 & 111 & -1816.31 & \pm 1883.96 \\
\hline \multirow[t]{2}{*}{3} & -7.27 & .15 & 112 & -13413.33 & 864.04 \\
\hline & & & 122 & -2399.29 & 751.44 \\
\hline$i j$ & $\mu_{i j}$ & & 222 & -20390.95 & 1406.62 \\
\hline 11 & 1091.77 & \pm 19.55 & 113 & -159.77 & 666.01 \\
\hline 12 & 121.87 & 9.98 & 123 & 339.50 & 388.35 \\
\hline 22 & 540.24 & 12.91 & 223 & -191.01 & 559.59 \\
\hline 13 & -15.31 & 7.61 & 133 & -659.45 & 453.92 \\
\hline 23 & 10.49 & 6.55 & 233 & -5667.18 & 474.23 \\
\hline 33 & 286.41 & 7.30 & 333 & -235.41 & 585.99 \\
\hline$i j k l$ & $\mu_{i j k l}$ & & $i j k l$ & $\kappa_{i j k l}$ & \\
\hline 1111 & 6363554.95 & \pm 318091.89 & 1111 & 2787687.85 & \pm 326572.65 \\
\hline 1112 & 398882.89 & 116713.07 & 1112 & -272.86 & 118957.05 \\
\hline 1113 & -146806.98 & 96850.45 & 1113 & -96666.77 & 97992.36 \\
\hline 1122 & 1362095.13 & 78776.76 & 1122 & 742572.73 & 82615.38 \\
\hline 1123 & 22168.02 & 37971.88 & 1123 & 14444.49 & 38708.40 \\
\hline 1133 & 784584.24 & 52065.51 & 1133 & 471419.86 & 53804.97 \\
\hline 1222 & 246216.17 & 88605.76 & 1222 & 48700.87 & 89422.91 \\
\hline 1223 & -27222.66 & 34791.88 & 1223 & -21509.60 & 35081.19 \\
\hline 1233 & 72597.64 & 27733.59 & 1233 & 38014.26 & 27986.78 \\
\hline 1333 & -13619.80 & 42830.96 & 1333 & -466.10 & 43014.61 \\
\hline 2222 & 2545140.85 & 218636.92 & 2222 & 1669556.37 & 219966.77 \\
\hline 2223 & -9405.17 & 67345.34 & 2223 & -26409.79 & 67645.47 \\
\hline 2233 & 580888.25 & 50011.35 & 2233 & 425936.06 & 50591.69 \\
\hline 2333 & -12789.16 & 47716.14 & 2333 & -21804.26 & 47835.90 \\
\hline 3333 & 802499.71 & 66491.61 & 3333 & 556403.52 & 66884.62 \\
\hline
\end{tabular}

Table 10. Central moments and cumulants for CNS3 heliocentric sample limited by $|\boldsymbol{V}|=40 \mathrm{~km} \mathrm{~s}^{-1}$, with 897 stars.

\begin{tabular}{|c|c|c|c|c|c|}
\hline $\bar{i}$ & $\overline{V_{i}}$ & & $i j k$ & $\mu_{i j k}$ & \\
\hline 1 & -4.59 & \pm .59 & & & \\
\hline 2 & -9.18 & .40 & 111 & 1229.92 & \pm 283.68 \\
\hline \multirow[t]{2}{*}{3} & -5.57 & .38 & 112 & 370.22 & 134.91 \\
\hline & & & 122 & 478.13 & 101.04 \\
\hline$i j$ & $\mu_{i j}$ & & 222 & 427.51 & 170.56 \\
\hline 11 & 307.29 & \pm 11.27 & 113 & 263.56 & 19.20 \\
\hline 12 & 33.48 & 6.38 & 123 & -112.09 & 72.48 \\
\hline 22 & 145.23 & 6.80 & 223 & 170.30 & 111.38 \\
\hline 13 & .04 & 6.30 & 133 & 282.21 & 106.07 \\
\hline 23 & 3.96 & 4.82 & 233 & 166.04 & 84.27 \\
\hline 33 & 129.17 & 6.75 & 333 & 404.25 & 135.78 \\
\hline$i j k l$ & $\mu_{i j k l}$ & & $i j k l$ & $\kappa_{i j k l}$ & \\
\hline 1111 & 208279.16 & \pm 13859.91 & 1111 & -75002.06 & \pm 18328.11 \\
\hline 1112 & 25430.61 & 5337.89 & 1112 & -5430.22 & 6700.69 \\
\hline 1113 & 5915.21 & 4406.77 & 1113 & 5874.66 & 5536.52 \\
\hline 1122 & 37665.03 & 2627.29 & 1122 & -9204.94 & 4598.58 \\
\hline 1123 & 1637.67 & 1704.74 & 1123 & 417.14 & 2306.23 \\
\hline 1133 & 35552.32 & 2605.56 & 1133 & -4141.73 & 4388.15 \\
\hline 1222 & 3204.61 & 2989.68 & 1222 & -11381.00 & 3596.88 \\
\hline 1223 & 1304.64 & 1363.12 & 1223 & 1032.96 & 1662.59 \\
\hline 1233 & 331.00 & 1461.10 & 1233 & -3993.64 & 1799.97 \\
\hline 1333 & -503.23 & 3235.48 & 1333 & -520.27 & 3528.99 \\
\hline 2222 & 62556.84 & 8574.23 & 2222 & -721.05 & 9231.34 \\
\hline 2223 & 1830.12 & 2583.89 & 2223 & 103.73 & 2873.83 \\
\hline 2233 & 20813.61 & 1908.45 & 2233 & 2021.79 & 2664.91 \\
\hline 2333 & 2071.27 & 2683.15 & 2333 & 535.77 & 2908.95 \\
\hline 3333 & 57608.70 & 6125.75 & 3333 & 7550.47 & 6830.80 \\
\hline
\end{tabular}


S. Alcobé and R. Cubarsi: Disk populations from HIPPARCOS kinematic data, Online Material p 4

Table 11. Central moments and cumulants for CNS3 heliocentric sample limited by $|\boldsymbol{V}|=135 \mathrm{~km} \mathrm{~s}^{-1}$, with 1,874 stars.

\begin{tabular}{|c|c|c|c|c|c|}
\hline $\bar{i}$ & $\overline{V_{i}}$ & & $i j k$ & $\mu_{i j k}$ & \\
\hline 1 & -10.52 & \pm 0.85 & & & \\
\hline 2 & -20.64 & 0.57 & 111 & 8876.25 & \pm 3406.31 \\
\hline \multirow[t]{2}{*}{3} & -7.86 & 0.46 & 112 & -5408.54 & 1348.51 \\
\hline & & & 122 & -2003.15 & 1007.97 \\
\hline$i j$ & $\mu_{i j}$ & & 222 & -12751.01 & 1518.78 \\
\hline 11 & 1351.75 & \pm 49.52 & 113 & -182.91 & 1059.98 \\
\hline 12 & 144.68 & 23.40 & 123 & -872.04 & 651.81 \\
\hline 22 & 609.54 & 26.22 & 223 & 245.21 & 720.99 \\
\hline 13 & -11.31 & 20.32 & 133 & -67.10 & 820.45 \\
\hline 23 & -3.02 & 15.21 & 233 & -2228.39 & 773.33 \\
\hline 33 & 396.37 & 19.97 & 333 & -32.55 & 1342.73 \\
\hline$i j k l$ & $\mu_{i j k l}$ & & $i j k l$ & $\kappa_{i j k l}$ & \\
\hline 1111 & 6423533.21 & \pm 514972.64 & 1111 & 941856.18 & \pm 564779.46 \\
\hline 1112 & 585108.65 & 148337.71 & 1112 & -1593.17 & 162852.12 \\
\hline 1113 & -54903.61 & 138022.29 & 1113 & -9054.92 & 146310.84 \\
\hline 1122 & 1047434.96 & 75808.21 & 1122 & 181621.52 & 100724.51 \\
\hline 1123 & -42884.09 & 44089.91 & 1123 & -35525.23 & 48922.00 \\
\hline 1133 & 773938.37 & 62729.44 & 1133 & 237886.31 & 78164.93 \\
\hline 1222 & 225710.01 & 76839.98 & 1222 & -38851.31 & 82962.41 \\
\hline 1223 & 26413.31 & 36545.39 & 1223 & 34179.78 & 38816.65 \\
\hline 1233 & 24774.37 & 31593.87 & 1233 & -32640.10 & 33857.17 \\
\hline 1333 & -24339.55 & 63496.37 & 1333 & -10895.36 & 65095.92 \\
\hline 2222 & 1659618.08 & 163083.29 & 2222 & 544986.71 & 172222.76 \\
\hline 2223 & -32602.98 & 62668.70 & 2223 & -27073.56 & 64727.60 \\
\hline 2233 & 433553.75 & 53532.41 & 2233 & 191928.92 & 58094.71 \\
\hline 2333 & -59220.13 & 63066.55 & 2333 & -55624.48 & 63942.37 \\
\hline 3333 & 904761.36 & 151700.94 & 3333 & 433427.48 & 154160.14 \\
\hline
\end{tabular}


S. Alcobé and R. Cubarsi: Disk populations from HIPPARCOS kinematic data, Online Material p 5

Table 12. Central moments and cumulants for CNS3 heliocentric sample limited by $|\boldsymbol{V}|=180 \mathrm{~km} \mathrm{~s}^{-1}$, with 1915 stars.

\begin{tabular}{|c|c|c|c|c|c|}
\hline $\bar{i}$ & $\overline{\overline{V_{i}}}$ & & $\overline{i j k}$ & $\overline{\overline{\mu_{i j k}}}$ & \\
\hline 1 & -11.45 & \pm .90 & & & \\
\hline 2 & -21.82 & .61 & 111 & 1309.94 & \pm 5171.06 \\
\hline \multirow[t]{2}{*}{3} & -7.89 & .48 & 112 & -10721.67 & 2083.96 \\
\hline & & & 122 & -3364.21 & 1701.10 \\
\hline$i j$ & $\mu_{i j}$ & & 222 & -20668.71 & 2700.51 \\
\hline 11 & 1540.75 & \pm 60.98 & 113 & 363.02 & 1701.32 \\
\hline 12 & 176.23 & 29.42 & 123 & -1167.35 & 1145.48 \\
\hline 22 & 718.23 & 34.46 & 223 & -1209.85 & 1373.29 \\
\hline 13 & -32.96 & 25.11 & 133 & -463.73 & 1234.83 \\
\hline 23 & .13 & 20.11 & 233 & -4552.48 & 1230.22 \\
\hline 33 & 448.26 & 23.38 & 333 & 103.41 & 1664.87 \\
\hline$i j k l$ & $\mu_{i j k l}$ & & $i j k l$ & $\kappa_{i j k l}$ & \\
\hline 1111 & 9496012.75 & \pm 889329.83 & 1111 & 2374280.91 & \pm 947023.58 \\
\hline 1112 & 774222.95 & 257767.71 & 1112 & -40361.94 & 275462.75 \\
\hline 1122 & 1688972.74 & 158797.21 & 1113 & -382109.69 & 247953.42 \\
\hline 1222 & 367630.44 & 164384.71 & 1122 & 520238.20 & 186603.42 \\
\hline 2222 & 2790107.92 & 348373.02 & 1123 & 9268.46 & 103853.96 \\
\hline 1113 & -534445.90 & 237724.51 & 1133 & 515423.35 & 133450.10 \\
\hline 1123 & -2148.25 & 98828.48 & 1222 & -12095.58 & 171005.50 \\
\hline 1223 & -63455.81 & 88772.96 & 1223 & -39830.47 & 90957.37 \\
\hline 2223 & 49508.22 & 148346.63 & 1233 & -12795.59 & 75430.32 \\
\hline 1133 & 1208247.43 & 117425.10 & 1333 & 5828.21 & 106339.50 \\
\hline 1233 & 66192.73 & 73411.24 & 2222 & 1242526.03 & 358769.24 \\
\hline 2233 & 774329.70 & 109298.75 & 2223 & 49229.30 & 150442.26 \\
\hline 1333 & -38491.60 & 104279.83 & 2233 & 452376.20 & 113954.90 \\
\hline 2333 & -114497.42 & 111341.34 & 2333 & -114671.49 & 112431.32 \\
\hline 3333 & 1247892.30 & 192332.12 & 3333 & 645089.73 & 195729.12 \\
\hline
\end{tabular}

